Pacific Journal of Mathematics

THE POWER 3/2 APPEARING IN THE ESTIMATE OF 


\title{
THE POWER 3/2 APPEARING IN THE ESTIMATE OF ANALYTIC CAPACITY
}

\section{TAKAFUMI MURAI}

\begin{abstract}
We show that the power $3 / 2$ appearing in the estimate of analytic capacity is best possible.
\end{abstract}

1. Introduction. For a compact set $E$ in the complex plane $\mathbb{C}$, $H^{\infty}\left(E^{c}\right)$ denotes the Banach space of bounded analytic functions in $E^{c}=\mathbb{C} \cup\{\infty\}-E$ with supremum norm $\|\cdot\|_{H^{\infty}}$. The analytic capacity of $E$ is defined by

$$
\gamma(E)=\sup \left\{\left|f^{\prime}(\infty)\right| ;\|f\|_{H^{\infty}} \leq 1, f \in H^{\infty}\left(E^{c}\right)\right\},
$$

where $f^{\prime}(\infty)=\lim _{z \rightarrow \infty} z(f(z)-f(\infty))$, i.e., $f^{\prime}(\infty)$ is the $(1 / z)$-coefficient of the Taylor expansion of $f(z)$ at infinity. It is easily seen that $\gamma(E) \leq|E|$, where $|E|$ is the (generalized) length of $E$; if $E$ is a subset of the real line $\mathbb{R}$, then $|E|$ equals its 1-dimension Lebesgue measure (cf. Garnett [4, Chap. III]). Vitushkin [12] constructed an example $Q_{\infty}$ such that $\gamma\left(Q_{\infty}\right)=0$ and $\left|Q_{\infty}\right|>0$ (cf. [4, p. 87]). Denjoy [3] showed that $\gamma(E)>0$ if $E$ is a subset of a rectifiable curve such that $|E|>0$. But his proof has a serious gap, and his theorem was, for a while, called the Denjoy conjecture. As is easily seen, we may assume that $E$ is a subset of a rectifiable graph. Let $\operatorname{pr} E$ denote the projection of $E$ to $\mathbb{R}$. Since pr is a contraction [6, p. 377], it is natural to try the lower estimate of $\gamma(E)$ by $\gamma(\operatorname{pr} E)$. Pommerenke [11] showed that $\gamma(\operatorname{pr} E)=|\operatorname{pr} E| / 4$. Hence this approach is equivalent to comparing $\gamma(E)$ with $|\operatorname{pr} E|$. To do this, the study of the Cauchy-Hilbert transform on $C^{1}$ graphs is necessary (Davie [2]). In 1977, Calderón [1] succeeded in proving its boundedness, and, using his theorem, Marshall [8] finally settled the Denjoy conjecture in the affirmative. After Marshall's theorem, we are concerned with studying further relations between $\gamma(E)$ and $|\operatorname{pr} E|$. Using an estimate of the Cauchy-Hilbert transform on Lipschitz graphs [10, p. 53], the author [9] showed that

$$
\gamma(E) \geq C_{0}|\operatorname{pr} E|^{3 / 2}
$$

if $E$ is a subset of a rectifiable graph $\Gamma$ satisfying $|\Gamma|=1$, where $C_{0}$ is an absolute constant. The main purpose of this paper is to show 
that the power $3 / 2$ is best possible. Our method gives a new approach to the computation of analytic capacity, and suggests that analytic capacity is related to the theory of fractals (Mandelbrot [7]).

For an integer $p \geq 2$, we put

$$
B_{p}(x)=\frac{1}{2 p}\left\{1-(-1)^{k}\right\} \quad\left(\frac{k}{p} \leq x<\frac{k+1}{p}, 0 \leq k \leq p-1\right) .
$$

For an $n$-tuple $\left(p_{1}, \ldots, p_{n}\right)$ of integers larger than or equal to 2 , we put

$$
A\left(x ; p_{1}, \ldots, p_{n}\right)=\sum_{j=1}^{n} B_{p_{1} \cdots p_{j}}(x) .
$$

A set $\Gamma \subset \mathbb{C}$ is called a crank of degree $n$ if it is expressed in the form

$$
\Gamma=\Gamma\left(p_{1}, \ldots, p_{n}\right)=\left\{x+i A\left(x ; p_{1}, \ldots, p_{n}\right) ; 0 \leq x<1\right\}
$$

for some $n$-tuple $\left(p_{1}, \ldots, p_{n}\right)$ of integers larger than or equal to 2 . (The class of cranks in this paper is smaller than a class defined in [10, Chap. III].) We shall show

THEOREM. For any $n \geq 1$, there exists a crank $\Gamma_{n}$ of degree $n$ such that

$$
\frac{1}{C_{1}} \frac{1}{\sqrt{n}} \leq \gamma\left(\Gamma_{n}\right) \leq C_{1} \frac{1}{\sqrt{n}},
$$

where $C_{1}$ is an absolute constant.

Once this theorem is established, we can deduce the exactness of the power $3 / 2$ as follows. Adding some segments (perpendicular to the $x$ axis) to $\Gamma_{n}$, we obtain an arc connecting 0 and 1 . Then the length of this arc is less than or equal to $n+1$. Hence we can define a rectifiable graph $\Gamma_{n}^{\prime}$ so that $\left|\Gamma_{n}^{\prime}\right| \leq 3 n,\left|\operatorname{pr} E_{n}^{\prime}\right| \geq 1 / 2$, where $E_{n}^{\prime}=\Gamma_{n} \cap \Gamma_{n}^{\prime}$. Then $\gamma\left(E_{n}^{\prime}\right) \leq \gamma\left(\Gamma_{n}\right) \leq C_{1} / \sqrt{n}$. Contracting $E_{n}^{\prime}, \Gamma_{n}^{\prime}$, we define $E_{n}^{\prime \prime}$, $\Gamma_{n}^{\prime \prime}$ so that $\left|\Gamma_{n}^{\prime \prime}\right|=1$. Then

$$
\begin{aligned}
\gamma\left(E_{n}^{\prime \prime}\right) & =\gamma\left(E_{n}^{\prime}\right) /\left|\Gamma_{n}^{\prime}\right| \leq \sqrt{3} C_{1}\left|\Gamma_{n}^{\prime}\right|^{-3 / 2} \\
& \leq 2^{3 / 2} \sqrt{3} C_{1}\left\{\left|\operatorname{pr} E_{n}^{\prime}\right| /\left|\Gamma_{n}^{\prime}\right|\right\}^{3 / 2}=2^{3 / 2} \sqrt{3} C_{1}\left|\operatorname{pr} E_{n}^{\prime \prime}\right|^{3 / 2},
\end{aligned}
$$

which shows that the power $3 / 2$ cannot be replaced by any number less than $3 / 2$.

To prove our theorem, it is necessary to investigate cranks carefully. In $\S 2$, we shall give a formula $((1)$ in Proposition 1$)$ to compute analytic capacity. Proposition 2 is a generalization of Garnett's example [4, p. 87], and will be used to prove our theorem. Using the 
method in the proof of the formula, we shall, in $\S 3$, give the proof of our theorem. In the last section, we shall give a new proof of Pommerenke's theorem [11] as another application of Proposition 1; our method shows how to construct the extremal functions.

2. A formula for the computation of $\gamma(\cdot)$. Let $L^{2}(\Gamma)$ denote the $L^{2}$ space of functions on a finite union $\Gamma$ of smooth arcs with respect to the length element $|d z|$. The norm is denoted by $\|\cdot\|_{L^{2}(\Gamma)}$. The Cauchy-Hilbert transform $\mathscr{H}_{\Gamma}$ from $L^{2}(\Gamma)$ to itself is defined by

$$
\mathscr{H}_{\Gamma} f(z)=\frac{1}{\pi} \mathrm{p} \cdot \mathrm{v} \cdot \int_{\Gamma} \frac{f(w)}{w-z}|d w|,
$$

where p.v. is the principal value. This is a bounded operator and the norm is denoted by $\left\|\mathscr{H}_{\Gamma}\right\|_{L^{2}(\Gamma), L^{2}(\Gamma)}$. An operator $\overline{\mathscr{H}}_{\Gamma}$ is defined by $\overline{\mathscr{H}}_{\Gamma} f=\overline{\mathscr{K}_{\Gamma} \bar{f}}$, and $\mathscr{C}_{\Gamma}$ is the identity operator. We show

Proposition 1. Let $\Gamma$ be a finite union of smooth arcs. Then, for any $0<\varepsilon<1 /\left\|\mathscr{H}_{\Gamma}\right\|_{L^{2}(\Gamma), L^{2}(\Gamma)}$,

(1) $\gamma(\Gamma)=\frac{1}{\pi}\left\{|\Gamma|+\sum_{m=0}^{\infty}\left(1-\varepsilon^{2}\right)^{m} \sum_{l=0}^{\infty} \varepsilon^{2 l+2} \frac{(l+1)(l+2) \cdots(l+m)}{m !} d_{2 l+2}\left(\mathscr{H}_{\Gamma}\right)\right\}$, where

$$
d_{2 l}\left(\mathscr{H}_{\Gamma}\right)=\int_{\Gamma}\left(\mathscr{H}_{\Gamma} \overline{\mathscr{H}}_{\Gamma}\right)^{l} 1|d z| \quad\left(l \geq 0,\left(\mathscr{H}_{\Gamma} \overline{\mathscr{H}}_{\Gamma}\right)^{0}=\mathscr{I}_{\Gamma}\right)
$$

and $(l+1) \cdots(l+m) / m !=1$ if $m=0$. (First $\sum_{l=0}^{\infty}$ is taken, and next $\sum_{m=0}^{\infty}$ is taken.) If $\left\|\mathscr{I}_{\Gamma}+\overline{\mathscr{H}}_{\Gamma} \mathscr{H}_{\Gamma}\right\|_{L^{2}(\Gamma), L^{2}(\Gamma)}<2$, then

$$
\gamma(\Gamma)=\frac{1}{\pi}\left\{|\Gamma|+\sum_{m=0}^{\infty} 2^{-m-1} \sum_{l=0}^{\infty}\left(\begin{array}{c}
m \\
l
\end{array}\right) d_{2 l+2}\left(\mathscr{H}_{\Gamma}\right)\right\},
$$

where $\left(\begin{array}{l}0 \\ 0\end{array}\right)=1$. If $\lim _{l \rightarrow \infty} d_{2 l}\left(\mathscr{H}_{\Gamma}\right)=0$, then

$$
\gamma(\Gamma)=\frac{1}{\pi} \sum_{l=0}^{\infty} d_{2 l}\left(\mathscr{H}_{\Gamma}\right) .
$$

This is a version of Garabedian's theorem [4, p. 22] to $\mathscr{H}_{\Gamma}$. Equality (3) is applicable to give a new proof of Pommerenke's theorem. (See $\S 4$.) Notice that $\mathscr{I}_{\mathbb{R}}+\overline{\mathscr{H}}_{\mathbb{R}} \mathscr{H}_{\mathbb{R}}=0$, where $\mathscr{H}_{\mathbb{R}}$ is the Hilbert transform on $\mathbb{R}$. Hence (2) is applicable to compact sets $\Gamma$ on a Lipschitz graph which is a small perturbation of $\mathbb{R}$. For any $M>0$, there exists a 
crank $\Gamma^{\prime}$ such that $d_{2}\left(\mathscr{H}_{\Gamma^{\prime}}\right) \geq M[10$, p. 84]. Then Cauchy-Schwarz' inequality yields that

$$
d_{2^{l}}\left(\mathscr{H}_{\Gamma^{\prime}}\right) \geq d_{2}\left(\mathscr{H}_{\Gamma^{\prime}}\right)^{2^{l-1}} \geq M^{2^{l-1}} \quad(l \geq 1) .
$$

Hence (1) is necessary in this case.

Proof of Proposition 1. Let

$$
\gamma^{*}\left(\mathscr{H}_{\Gamma}\right)=\inf \left\{\left\|1+\mathscr{H}_{\Gamma} h\right\|_{L^{2}(\Gamma)}^{2}+\|h\|_{L^{2}(\Gamma)}^{2} ; h \in L^{2}(\Gamma)\right\} .
$$

We begin by showing that

$$
\gamma(\Gamma)=\frac{1}{\pi} \gamma^{*}\left(\mathscr{H}_{\Gamma}\right)
$$

For a compact set $E$ bounded by a finite number of smooth Jordan curves, we have

(6) $\gamma(E)=\frac{1}{2 \pi} \inf \left\{\int_{\partial E}|g(z)|^{2}|d z| ; g(\infty)=1, g\right.$ is analytic in $\left.E^{c}\right\}$

[4, p. 22]. Hence a standard argument yields that (6) holds with $E$ replaced by $\Gamma$; in this case, the boundary $\partial \Gamma$ has two sides. We define a smooth curve $\mathscr{L}$ tending to infinity so that $\Gamma \subset \mathscr{L}$ and that $\mathscr{L}=\mathbb{R}$ outside a large disk. Then $\mathscr{L}$ divides $\mathbb{C}$ into two domains $\Omega_{ \pm}$. For an analytic function $g(z)$ in $\Gamma^{c}$ such that $g(\infty)=1$ and $\int_{\partial \Gamma}|g(z)|^{2}|d z|<\infty$, we can write

$$
g(z)=1+\frac{1}{\pi} \int_{\Gamma} \frac{h(w)}{w-z} d w
$$

where the orientation of $d w$ is chosen so that $\Omega_{+}$lies to the left. Let $g_{ \pm}(z)$ be the nontangential limits of $g$ at $z \in \Gamma$ with respect to $\Omega_{ \pm}$, respectively. Then

$$
\begin{aligned}
g_{+}(z) & =1+\frac{1}{\pi} \mathbf{p} \cdot \mathbf{v} \cdot \int_{\Gamma} \frac{h(w)}{w-z} d w+i h(z) \\
& =1+\mathscr{H}_{\Gamma}(h \psi)(z)+i h(z) \quad(z \in \Gamma),
\end{aligned}
$$

where $\psi(z)=d z /|d z|$. Analogously,

$$
g_{-}(z)=1+\mathscr{H}_{\Gamma}(h \psi)(z)-i h(z) \quad(z \in \Gamma) .
$$

Thus

$$
\begin{aligned}
\int_{\partial \Gamma}|g(z)|^{2}|d z|=\left\|g_{+}\right\|_{L^{2}(\Gamma)}^{2}+\left\|g_{-}\right\|_{L^{2}(\Gamma)}^{2} \\
=\left\|1+\mathscr{H}_{\Gamma}(h \psi)+i h\right\|_{L^{2}(\Gamma)}^{2}+\left\|1+\mathscr{H}_{\Gamma}(h \psi)-i h\right\|_{L^{2}(\Gamma)}^{2} \\
\quad=2\left\{\left\|1+\mathscr{H}_{\Gamma}(h \psi)\right\|_{L^{2}(\Gamma)}^{2}+\|h\|_{L^{2}(\Gamma)}^{2}\right\} \\
\quad=2\left\{\left\|1+\mathscr{H}_{\Gamma}(h \psi)\right\|_{L^{2}(\Gamma)}^{2}+\|h \psi\|_{L^{2}(\Gamma)}^{2}\right\}
\end{aligned}
$$


because $|\psi(z)|=1 \quad(z \in \Gamma)$. This shows that the quantity in the right-hand side of $(6)(E=\Gamma)$ equals $\frac{1}{\pi} \gamma^{*}\left(\mathscr{H}_{\Gamma}\right)$, i.e., (5) holds.

We next compute $\gamma^{*}\left(\mathscr{H}_{\Gamma}\right)$. Fatou's lemma shows that there exists $h_{\Gamma} \in L^{2}(\Gamma)$ which attains the infimum in (4). A variational method yields that $\left(1+\mathscr{H}_{\Gamma} h_{\Gamma}, \mathscr{H}_{\Gamma} h\right)+\left(h_{\Gamma}, h\right)=0$ for all $h \in L^{2}(\Gamma)$, where $(\cdot, \cdot)$ is the (complex) inner product with respect to $|d z|$. Since the adjoint operator of $\mathscr{H}_{\Gamma}$ is $-\overline{\mathscr{H}}_{\Gamma}$, this shows that

$$
\left(\mathscr{I}_{\Gamma}-\overline{\mathscr{H}}_{\Gamma} \mathscr{H}_{\Gamma}\right) h_{\Gamma}=\overline{\mathscr{H}}_{\Gamma} 1 \text {. }
$$

Suppose that $h_{\Gamma}^{\prime} \in L^{2}(\Gamma)$ also attains the infimum in (4). Then $h_{\Gamma}^{\prime}$ satisfies (7), and hence

$$
\begin{aligned}
0 & =\left(\left(\mathscr{J}_{\Gamma}-\overline{\mathscr{H}}_{\Gamma} \mathscr{H}_{\Gamma}\right)\left(h_{\Gamma}-h_{\Gamma}^{\prime}\right), h_{\Gamma}-h_{\Gamma}^{\prime}\right) \\
& =\left\|h_{\Gamma}-h_{\Gamma}^{\prime}\right\|_{L^{2}(\Gamma)}^{2}+\left\|\mathscr{H}_{\Gamma}\left(h_{\Gamma}-h_{\Gamma}^{\prime}\right)\right\|_{L^{2}(\Gamma)}^{2} .
\end{aligned}
$$

This shows that $h_{\Gamma}^{\prime}=h_{\Gamma}$. Thus $h_{\Gamma}$ is uniquely determined. By (7), we have

$$
\begin{aligned}
\gamma^{*}\left(\mathscr{H}_{\Gamma}\right) & =\left\|1+\mathscr{H}_{\Gamma} h_{\Gamma}\right\|_{L^{2}(\Gamma)}^{2}+\left\|h_{\Gamma}\right\|_{L^{2}(\Gamma)}^{2} \\
& =\left(1+\mathscr{H}_{\Gamma} h_{\Gamma}, 1\right)+\left(\left(\mathscr{C}_{\Gamma}-\overline{\mathscr{H}}_{\Gamma} \mathscr{H}_{\Gamma}\right) h_{\Gamma}-\overline{\mathscr{H}}_{\Gamma} 1, h_{\Gamma}\right) \\
& =\int_{\Gamma}\left\{1+\mathscr{H}_{\Gamma} h_{\Gamma}\right\}|d z| .
\end{aligned}
$$

Let

$$
T_{\Gamma}=\left(\mathscr{I}_{\Gamma}-\varepsilon^{2 \overline{\mathscr{H}}_{\Gamma}} \mathscr{H}_{\Gamma}\right)^{-1}
$$

Then we can write

$$
T_{\Gamma}=\sum_{l=0}^{\infty} \varepsilon^{2 l}\left(\overline{\mathscr{H}}_{\Gamma}^{\mathscr{H}_{\Gamma}}\right)^{l}
$$

because $0<\varepsilon<1 /\left\|\mathscr{H}_{\Gamma}\right\|_{L^{2}(\Gamma), L^{2}(\Gamma)}$. We have, for any $h \in L^{2}(\Gamma)$,

$$
\begin{aligned}
& \left\|T_{\Gamma} h\right\|_{L^{2}(\Gamma)}^{2} \leq\left\|T_{\Gamma} h\right\|_{L^{2}(\Gamma)}^{2}+\varepsilon^{2}\left\|\mathscr{H}_{\Gamma} T_{\Gamma} h\right\|_{L^{2}(\Gamma)}^{2} \\
& \quad=\left(\left(\mathscr{C}_{\Gamma}-\varepsilon^{\left.2 \bar{H}_{\Gamma} \mathscr{H}_{\Gamma}\right)} T_{\Gamma} h, T_{\Gamma} h\right)=\left(h, T_{\Gamma} h\right) \leq\|h\|_{L^{2}(\Gamma)}\left\|T_{\Gamma} h\right\|_{L^{2}(\Gamma)},\right.
\end{aligned}
$$

which shows that $\left\|T_{\Gamma}\right\|_{L^{2}(\Gamma), L^{2}(\Gamma)} \leq 1$. Equality (7) can be rewritten as

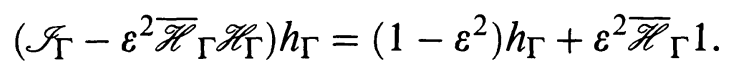

Observing this equality, we inductively define $\left(h_{m}\right)_{m=0}^{\infty}$ by $h_{0}=0$,

$$
h_{m}=T_{\Gamma}\left\{\left(1-\varepsilon^{2}\right) h_{m-1}+\varepsilon^{2} \overline{\mathscr{H}}_{\Gamma} 1\right\} \quad(m \geq 1) .
$$


Then

$$
\begin{aligned}
\left\|h_{m+1}-h_{m}\right\|_{L^{2}(\Gamma)} & =\left(1-\varepsilon^{2}\right)\left\|T_{\Gamma}\left(h_{m}-h_{m-1}\right)\right\|_{L^{2}(\Gamma)} \\
& \leq\left(1-\varepsilon^{2}\right)\left\|h_{m}-h_{m-1}\right\|_{L^{2}(\Gamma)} .
\end{aligned}
$$

Hence $\lim _{m \rightarrow \infty} h_{m}$ exists and satisfies (9), i.e., (7). Thus $h_{\Gamma}=$ $\lim _{m \rightarrow \infty} h_{m}$. Since

$$
\begin{aligned}
h_{m+1}-h_{m} & =\left(1-\varepsilon^{2}\right) T_{\Gamma}\left(h_{m}-h_{m-1}\right)=\cdots=\left(1-\varepsilon^{2}\right)^{m} T_{\Gamma}^{m} h_{1} \\
& =\varepsilon^{2}\left(1-\varepsilon^{2}\right)^{m} T_{\Gamma}^{m+1} \overline{\mathscr{H}}_{\Gamma} 1,
\end{aligned}
$$

we have

$$
h_{\Gamma}=\sum_{m=0}^{\infty}\left(h_{m+1}-h_{m}\right)=\varepsilon^{2} \sum_{m=0}^{\infty}\left(1-\varepsilon^{2}\right)^{m} T_{\Gamma}^{m+1 \overline{\mathscr{H}}_{\Gamma}} 1 .
$$

Consequently, (8) yields that

$$
\begin{aligned}
\gamma^{*}\left(\mathscr{H}_{\Gamma}\right) & =|\Gamma|+\sum_{m=0}^{\infty}\left(1-\varepsilon^{2}\right)^{m} \int_{\Gamma} \varepsilon^{2} \mathscr{H}_{\Gamma} T_{\Gamma}^{m+1} \overline{\mathscr{H}}_{\Gamma} 1|d z| \\
= & |\Gamma|+\sum_{m=0}^{\infty}\left(1-\varepsilon^{2}\right)^{m} \int_{\Gamma} \varepsilon^{2} \mathscr{H}_{\Gamma}\left\{\sum_{l=0}^{\infty} \varepsilon^{2 l}\left(\overline{\mathscr{H}}_{\Gamma} \mathscr{H}_{\Gamma}\right)^{l}\right\}^{m+1} \overline{\mathscr{H}}_{\Gamma} 1|d z| \\
= & |\Gamma|+\sum_{m=0}^{\infty}\left(1-\varepsilon^{2}\right)^{m} \sum_{l=0}^{\infty} \varepsilon^{2 l+2} \frac{(l+1) \cdots(l+m)}{m !} \int_{\Gamma}\left(\mathscr{H}_{\Gamma} \overline{\mathscr{H}}_{\Gamma}\right)^{l+1} 1|d z| \\
& =|\Gamma|+\sum_{m=0}^{\infty}\left(1-\varepsilon^{2}\right)^{m} \sum_{l=0}^{\infty} \varepsilon^{2 l+2} \frac{(l+1) \cdots(l+m)}{m !} d_{2 l+2}\left(\mathscr{H}_{\Gamma}\right) .
\end{aligned}
$$

Using (5), we obtain (1).

We can write

$$
\mathscr{I}_{\Gamma}-\overline{\mathscr{H}}_{\Gamma} \mathscr{H}_{\Gamma}=2\left\{\mathscr{I}_{\Gamma}-\frac{1}{2}\left(\mathscr{I}_{\Gamma}+\overline{\mathscr{H}}_{\Gamma} \mathscr{H}_{\Gamma}\right)\right\}
$$

Hence, if $\left\|\mathscr{C}_{\Gamma}+\overline{\mathscr{H}}_{\Gamma} \mathscr{H}_{\Gamma}\right\|_{L^{2}(\Gamma), L^{2}(\Gamma)}<2$, then

$$
h_{\Gamma}=\sum_{m=0}^{\infty} 2^{-m}\left(\mathscr{C}_{\Gamma}+\overline{\mathscr{H}}_{\Gamma} \mathscr{H}_{\Gamma}\right)^{m}\left(\frac{1}{2} \overline{\mathscr{H}}_{\Gamma}\right) 1 .
$$

Thus (5) and (8) yield (2).

Equality (7) shows that $\mathscr{H}_{\Gamma} h_{\Gamma}=\mathscr{H}_{\Gamma} \overline{\mathscr{H}}_{\Gamma} 1+\mathscr{H}_{\Gamma} \overline{\mathscr{H}}_{\Gamma} \mathscr{H}_{\Gamma} h_{\Gamma}$, and hence, by $(8)$,

$$
\gamma^{*}\left(\mathscr{H}_{\Gamma}\right)=\int_{\Gamma}\left\{1+\mathscr{H}_{\Gamma} \overline{\mathscr{H}}_{\Gamma} 1+\mathscr{H}_{\Gamma} \overline{\mathscr{H}}_{\Gamma} \mathscr{H}_{\Gamma} h_{\Gamma}\right\}|d z| .
$$


Repeating this argument, we have

$$
\begin{aligned}
\gamma^{*}\left(\mathscr{H}_{\Gamma}\right) & =\int_{\Gamma}\left\{\sum_{l=0}^{L}\left(\mathscr{H}_{\Gamma} \overline{\mathscr{H}}_{\Gamma}\right)^{l} 1+\mathscr{H}_{\Gamma}\left(\overline{\mathscr{H}}_{\Gamma} \mathscr{H}_{\Gamma}\right)^{L} h_{\Gamma}\right\}|d z| \\
& =\sum_{l=0}^{L} d_{2 l}\left(\mathscr{H}_{\Gamma}\right)-\int_{\Gamma}\left\{\left(\mathscr{H}_{\Gamma} \overline{\mathscr{H}}_{\Gamma}\right)^{L} \mathscr{H}_{\Gamma}\right\} 1(z) h_{\Gamma}(z)|d z| .
\end{aligned}
$$

If $\lim _{L \rightarrow \infty} d_{2 L}\left(\mathscr{H}_{\Gamma}\right)=0$, then

$$
\begin{aligned}
& \lim _{L \rightarrow \infty}\left|\int_{\Gamma}\left\{\left(\mathscr{H}_{\Gamma} \overline{\mathscr{H}}_{\Gamma}\right)^{L} \mathscr{H}_{\Gamma}\right\} 1(z) h_{\Gamma}(z)\right| d z|| \\
& \leq \lim _{L \rightarrow \infty}\left\|\left(\mathscr{H}_{\Gamma} \overline{\mathscr{H}}_{\Gamma}\right)^{L} \mathscr{H}_{\Gamma} 1\right\|_{L^{2}(\Gamma)}\left\|h_{\Gamma}\right\|_{L^{2}(\Gamma)} \\
& =\lim _{L \rightarrow \infty} d_{4 L+2}\left(\mathscr{H}_{\Gamma}\right)^{1 / 2}\left\|h_{\Gamma}\right\|_{L^{2}(\Gamma)}=0 .
\end{aligned}
$$

Hence (5) gives (3). This completes the proof of our proposition.

We now give a remark. There exists an analytic function $g_{\Gamma}(z)$ in $\Gamma^{c}$ such that $g_{\Gamma}(\infty)=1$ and $\gamma(\Gamma)=(1 / 2 \pi) \int_{\partial \Gamma}\left|g_{\Gamma}(z)\right||d z|$ [4, p. 19]. This is called the Garabedian function of $\Gamma$. Equality (5) shows that

$$
g_{\Gamma}(z)=\left\{1+\frac{1}{\pi} \int_{\Gamma} \frac{h_{\Gamma}(w)}{w-z}|d w|\right\}^{2} .
$$

There exists $f_{\Gamma} \in H^{\infty}\left(\Gamma^{c}\right)$ such that $\left\|f_{\Gamma}\right\|_{H^{\infty}}=1$ and $f_{\Gamma}^{\prime}(\infty)=\gamma(\Gamma)$ [4, p. 18]. This is called the Ahlfors function of $\Gamma$. We have

$$
f_{\Gamma}(z)=\frac{-\frac{1}{\pi}\left\{\int_{\Gamma} \frac{|d w|}{w-z}+\int_{\Gamma} \frac{\overline{\mathscr{H}}_{\Gamma} \bar{h}_{\Gamma}(w)}{w-z}|d w|\right\}}{\left\{1+\frac{1}{\pi} \int_{\Gamma} \frac{h_{\Gamma}(w)}{w-z}|d w|\right\}}
$$

To see this, let $f(z)$ denote the function in the right-hand side. Since $g_{\Gamma}(z)$ does not take 0 in $\Gamma^{c}, f(z)$ is analytic in $\Gamma^{c}[\mathbf{4}$, p. 21]. We have $f^{\prime}(\infty)=\frac{1}{\pi} \gamma^{*}\left(\mathscr{H}_{\Gamma}\right)=\gamma(\Gamma)$ and

$$
f_{ \pm}(z)=-\frac{\mathscr{H}_{\Gamma} 1(z) \pm i \tilde{\psi}(z)+\mathscr{H}_{\Gamma} \overline{\mathscr{H}}_{\Gamma} \bar{h}_{\Gamma}(z) \pm i \overline{\mathscr{H}}_{\Gamma} \bar{h}_{\Gamma}(z) \tilde{\psi}(z)}{1+\mathscr{H}_{\Gamma} h_{\Gamma}(z) \pm i h_{\Gamma}(z) \tilde{\psi}(z)},
$$

where $\tilde{\psi}(z)=|d z| / d z$ and $f_{ \pm}(z)$ are the nontangential limits of $f$ at $z \in \Gamma$ with respect to $\Omega_{ \pm}$, respectively. Equality (7) shows that

$$
\begin{aligned}
\mathscr{H}_{\Gamma} 1+ & i \tilde{\psi}+\mathscr{H}_{\Gamma} \overline{\mathscr{H}}_{\Gamma} \bar{h}_{\Gamma}+i\left(\overline{\mathscr{H}}_{\Gamma} \bar{h}_{\Gamma}\right) \tilde{\psi} \\
& =\mathscr{H}_{\Gamma} 1+i \tilde{\psi}+\left(\bar{h}_{\Gamma}-\mathscr{H}_{\Gamma} 1\right)+i\left(\overline{\mathscr{H}}_{\Gamma} \bar{h}_{\Gamma}\right) \tilde{\psi} \\
& =i \tilde{\psi}+i\left(\overline{\mathscr{H}}_{\Gamma} \bar{h}_{\Gamma}\right) \tilde{\psi}+\bar{h}_{\Gamma}=i \tilde{\psi}\left\{\overline{1+\mathscr{H}_{\Gamma} h_{\Gamma}+i h_{\Gamma} \tilde{\psi}}\right\},
\end{aligned}
$$


which yields that $\left|f_{+}(z)\right|=1$ on $\Gamma$. Analogously, $\left|f_{-}(z)\right|=1$ on $\Gamma$. Thus $\|f\|_{H^{\infty}}=1$. This shows that $f=f_{\Gamma}$.

For the proof of our theorem, we note

Proposition 2. Let $0<\delta_{0}<1$ and let $\left(q_{n}\right)_{n=1}^{\infty}$ be a sequence of integers larger than or equal to 2 such that

$$
\sum_{n=j}^{\infty}\left(q_{j} \cdots q_{n}\right)^{-1} \leq \delta_{0} \quad(j \geq 1)
$$

Then

$$
\lim _{n \rightarrow \infty} \sup \gamma\left(\Gamma\left(p_{1}, \ldots, p_{n}\right)\right)=0,
$$

where the supremum is taken over all $n$-tuples $\left(p_{1}, \cdots, p_{n}\right)$ satisfying $p_{j} \geq q_{j} \quad(1 \leq j \leq n)$.

This is a generalization of Garnett's example [4, p. 87], and used later. Notice that $\sum_{n=1}^{\infty} 2^{-n}=1$. A sequence $\left(\Gamma\left(\mathbf{2}_{n}\right)\right)_{n=1}^{\infty}\left(\mathbf{2}_{n}\right.$ is the $n$ tuple of 2) topologically converges to a segment $\{x+i x ; 0 \leq x<1\}$, and these cranks behave like cranks of degree 1 with respect to this segment. Hence we have $\lim \sup _{n \rightarrow \infty} \gamma\left(\Gamma\left(\mathbf{2}_{n}\right)\right)>0$. This shows that our proposition is sharp in a sense. Since a minor change of the argument in [10, p. 81] yields the required equality, we omit the proof (cf. Jones [5]).

3. Proof of Theorem. In this section, we give the proof of our theorem. Let $L^{q}$ denote the $L^{q}$ space of functions on $[0,1)$ with respect to the 1-dimension Lebesgue measure $|\cdot|(1 \leq q<\infty)$. For a kernel $K=K(x, y)$ on $[0,1) \times[0,1)$, we simply write by the same notation $K$ an operator defined by this kernel, and write by $\bar{K}$ an operator defined by $\overline{K(x, y)} ;\|K\|_{L^{q}, L^{q^{\prime}}}$ denotes the norm of $K$ as an operator from $L^{q}$ to $L^{q^{\prime}}$. The identity operator is denoted by Id. A kernel $K$ is anti-symmetric if $K(x, y)=-K(y, x)(x \neq y)$. A kernel $K$ is of type 0 if

$$
\sup _{x, y \in[0,1)}\left\{|K(x, y)|+\left|\frac{\partial}{\partial x} K(x, y)\right|+\left|\frac{\partial}{\partial y} K(x, y)\right|\right\}<\infty .
$$

A kernel $K$ is of type 1 if $\|K\|_{L^{4}, L^{4}}<\infty$ and if there exists a sequence $\left(K_{j}\right)_{j=1}^{\infty}$ of kernels of type 0 such that

$$
\lim _{j \rightarrow \infty}\left\|K_{j}-K\right\|_{L^{4}, L^{2}}=0, \quad \sup _{j \geq 1}\left\|K_{j}\right\|_{L^{4}, L^{4}}<\infty .
$$


Kernels used in this section are bounded as operators from $L^{q}$ to itself for all $1<q<\infty$. Let

$$
\begin{aligned}
& \gamma^{*}(K)=\inf \left\{\|1+K h\|_{L^{2}}^{2}+\|h\|_{L^{2}}^{2} ; h \in L^{2}\right\}, \\
& d_{2 l}(K)=\int_{0}^{1}(K \bar{K})^{l} 1 d x \quad\left(l \geq 0, \quad(K \bar{K})^{0}=\mathrm{Id}\right) .
\end{aligned}
$$

Recall the function $A\left(x ; p_{1}, \ldots, p_{n}\right)$ in the introduction. Let

$$
H(x, y)=\mathscr{H}_{\mathbb{R}}(x, y)=\frac{1}{\pi} \frac{1}{y-x},
$$

$$
\begin{aligned}
& H\left[p_{1}, \ldots, p_{n}\right](x, y) \\
& \quad=\frac{1}{\pi} \frac{1}{(y-x)+i\left(A\left(y ; p_{1}, \ldots, p_{n}\right)-A\left(x ; p_{1}, \ldots, p_{n}\right)\right)}, \\
& \Delta\left[p_{1}, \ldots, p_{n}\right]=H\left[p_{1}, \ldots, p_{n}\right]-H\left[p_{1}, \ldots, p_{n-1}\right] \quad(n \geq 1),
\end{aligned}
$$

where $H\left[p_{1}, \ldots, p_{n-1}\right]=H$ if $n=1$. Then

$$
H\left[p_{1}, \ldots, p_{n}\right]=H+\sum_{j=1}^{n} \Delta\left[p_{1}, \ldots, p_{j}\right] .
$$

Since all components/segments of $\Gamma\left(p_{1}, \cdots, p_{n}\right)$ are parallel to

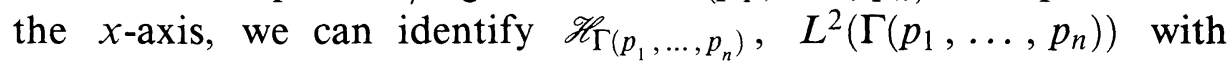
$H\left[p_{1}, \ldots, p_{n}\right], L^{2}$, respectively. We have $\left\|H\left[p_{1}, \ldots, p_{n}\right]\right\|_{L^{2}, L^{2}} \leq$ $C_{2} \sqrt{n}$ for some absolute constant $C_{2}[\mathbf{1 0}$, p. 84]. Hence Proposition 1 shows that

$$
\begin{aligned}
\gamma( & \left.\Gamma\left(p_{1}, \ldots, p_{n}\right)\right)=\frac{1}{\pi} \gamma^{*}\left(H\left[p_{1}, \ldots, p_{n}\right]\right) \\
=\frac{1}{\pi}\{1 & +\sum_{m=0}^{\infty}\left(1-\varepsilon_{n}^{2}\right)^{m} \\
& \left.\times \sum_{l=0}^{\infty} \varepsilon_{n}^{2 l+2} \frac{(l+1) \cdots(l+m)}{m !} d_{2 l+2}\left(H\left[p_{1}, \ldots, p_{n}\right]\right)\right\},
\end{aligned}
$$

where $\varepsilon_{n}=\left(2 C_{2} \sqrt{n}\right)^{-1}$. We shall inductively estimate

$$
\lim _{p_{1} \rightarrow \infty} \cdots \lim _{p_{n} \rightarrow \infty} \gamma^{*}\left(H\left[p_{1}, \ldots, p_{n}\right]\right)
$$

where $\lim _{p_{n} \rightarrow \infty}$ is taken first and $\lim _{p_{1} \rightarrow \infty}$ is taken last. For $E \subset \mathbb{R}$, $\chi_{E}$ denotes its characteristic function, and, for $x \in \mathbb{R}, l(x)$ denotes its integral part. Here are some lemmas necessary for the estimate. 
LemMa 3. For two kernels $K$ and $K^{\prime}$,

$$
\gamma^{*}\left(K+K^{\prime}\right) \leq 2\left(1+\left\|K^{\prime}\right\|_{L^{2}, L^{2}}^{2}\right) \gamma^{*}(K) .
$$

Proof. We have, for any $h \in L^{2}$,

$$
\left\|1+\left(K+K^{\prime}\right) h\right\|_{L^{2}}^{2}+\|h\|_{L^{2}}^{2} \leq 2\left(1+\left\|K^{\prime}\right\|_{L^{2}, L^{2}}^{2}\right)\left\{\|1+K h\|_{L^{2}}^{2}+\|h\|_{L^{2}}^{2}\right\},
$$

which yields the required inequality.

LEMMA 4. Let $K$ be an anti-symmetric kernel such that

$$
\lim _{l \rightarrow \infty} d_{2 l}(K)=0 \text {. }
$$

Then

$$
\gamma^{*}(K)=\sum_{l=0}^{\infty} d_{2 l}(K)
$$

Since this is a version of (3) to $K$, we omit the proof.

LEMMA 5. For an anti-symmetric kernel $K, 0<\varepsilon_{0} \leq\left(3\|K\|_{L^{2}, L^{2}}\right)^{-1}$ and $w \in U=\{\zeta \in \mathbb{C} ;|\zeta|<2,|\arg \zeta|<\pi / 4\}$,

$$
\begin{array}{r}
1+\sum_{m=0}^{\infty}\left(1-\varepsilon_{0}^{2}\right)^{m} \sum_{l=0}^{\infty} w^{2 l+2} \varepsilon_{0}^{2 l+2} \frac{(l+1) \cdots(l+m)}{m !} d_{2 l+2}(K) \\
\left(=\gamma^{*}(w ; K), \text { say }\right)
\end{array}
$$

exists and $\gamma^{*}(w ; K)$ is analytic in $U$.

Proof. Let

$$
T(w ; K)=\left(\operatorname{Id}-w^{2} \varepsilon_{0}^{2} \bar{K} K\right)^{-1}
$$

Then

$$
\begin{aligned}
w^{2} \varepsilon_{0}^{2} & \int_{0}^{1} K T(w ; K)^{m+1} \bar{K} 1 d x \\
& =\sum_{l=0}^{\infty} w^{2 l+2} \varepsilon_{0}^{2 l+2} \frac{(l+1) \cdots(l+m)}{m !} d_{2 l+2}(K)
\end{aligned}
$$

because $2 \varepsilon_{0}\|K\|_{L^{2}, L^{2}}<1$. Evidently, this is analytic in $U$. Since $K$ is anti-symmetric and $\operatorname{Re} w^{2}>0 \quad\left(w \in U, \operatorname{Re} w^{2}\right.$ is the real part of $w^{2}$ ), we have, in the same manner as in the proof of (1),

$$
\|T(w ; K)\|_{L^{2}, L^{2}} \leq 1 \quad(w \in U) .
$$


Thus the convergence of $\sum_{m=0}^{\infty}$ in (13) is uniform in $U$, which shows that $\gamma^{*}(w ; K)$ exists and is analytic in $U$.

Lemma 6. For any $l \geq 0$,

$$
\lim _{p \rightarrow \infty} d_{2 l}(\Delta[p]) \quad\left(=d_{2 l}(\Delta[\infty]), \text { say }\right)
$$

exists and

$$
d_{2}(\Delta[\infty]) \leq-\frac{1}{25 \pi^{2}}
$$

Proof. We put

$$
\begin{aligned}
R(s, t)= & \frac{1}{\pi}\left\{\frac{1}{t-s+1+i}-\frac{1}{t-s+1}\right\} \\
& -\frac{2}{\pi} \sum_{m=1}^{\infty}\left\{\frac{t-s+1+i}{4 m^{2}-(t-s+1+i)^{2}}-\frac{t-s+1}{4 m^{2}-(t-s+1)^{2}}\right\}
\end{aligned}
$$

and show that

$$
\lim _{p \rightarrow \infty} d_{2 l}(\Delta[p])=\frac{1}{2} \int_{0}^{1}\left\{R^{2 l} 1+\bar{R}^{2 l} 1\right\} d s .
$$

Let

$$
\begin{gathered}
W_{p}=\bigcup_{m, \text { odd }}\left[\frac{m}{p}, \frac{m+1}{p}\right), \quad W_{p}^{\prime}=\bigcup_{m, \text { even }}\left[\frac{m}{p}, \frac{m+1}{p}\right), \\
X_{p}=\bigcup_{m=l(\log p)}^{p-l(\log p)-1}\left[\frac{m}{p}, \frac{m+1}{p}\right), \\
s_{x}=p x-l(p x) \quad(0 \leq x<1, p \geq 2) .
\end{gathered}
$$

Notice that $\left|[0,1)-X_{p}\right| \leq 2 \quad l(\log p) / p$ and $\|\Delta[p]\|_{L^{4}, L^{4}} \leq 10$. Since $\Delta[p](x, y)=0 \quad\left(x, y \in W_{p} ; x, y \in W_{p}^{\prime}\right)$, we have

$$
\begin{aligned}
d_{2 l}(\Delta[p])= & \int_{0}^{1}(\Delta[p] \overline{\Delta[p]})^{l-1} \Delta[p]\left\{\chi_{W_{p}} \overline{\Delta[p]} \chi_{W_{p}^{\prime}}+\chi_{W_{p}^{\prime}} \overline{\Delta[p]} \chi_{W_{p}}\right\} d x \\
= & \int_{0}^{1}(\Delta[p] \overline{\Delta[p]})^{l-1} \Delta[p]\left\{\chi_{W_{p} \cap X_{p}} \overline{\Delta[p]} \chi_{W_{p}^{\prime}}+\chi_{W_{p}^{\prime} \cap X_{p}} \overline{\Delta[p]} \chi_{W_{p}}\right\} d x \\
& +O\left(\left(\frac{\log p}{p}\right)^{1 / 4}\right) .
\end{aligned}
$$

We now study $\overline{\Delta[p]} \chi_{W_{p}^{\prime}}(x) \quad\left(x \in W_{p} \cap X_{p}\right)$. Without loss of generality, we may assume that $p$ is even. Since $x \in W_{p} \cap X_{p}, l(p x)$ is even and 
$l(\log p) \leq l(p x) \leq p-\imath(\log p)-1$. We may assume that $l(\log p) \leq$ $\imath(p x) \leq p / 2$. We have

$$
\begin{aligned}
\overline{\Delta[p]} \chi_{W_{p}^{\prime}}(x)=\frac{1}{\pi} \int_{W_{p}^{\prime}}\left\{\frac{1}{y-x-i / p}-\frac{1}{y-x}\right\} d y \\
=\frac{1}{\pi} \int_{W_{p}}\left\{\frac{1}{y-x+1 / p-i / p}-\frac{1}{y-x+1 / p}\right\} d y \\
=\frac{1}{\pi} \sum_{m=0}^{(p / 2)-1} \int_{0}^{1 / p}\left\{\frac{1}{(2 m / p+y)-(l(p x) / p+x-l(p x) / p)+1 / p-i / p}\right. \\
=\frac{1}{\pi} \sum_{m=0}^{l(p x)}+\frac{1}{\pi} \sum_{m=l(p x)+1}^{(p / 2)-1}=L_{1}+L_{2},
\end{aligned}
$$

$$
\begin{aligned}
L_{1}= & \frac{1}{\pi} \int_{0}^{1}\left\{\frac{1}{t-s_{x}+1-i}-\frac{1}{t-s_{x}+1}\right\} d t+\frac{1}{\pi} \sum_{0 \leq m \leq l(p x), m \neq l(p x) / 2} \\
= & \frac{1}{\pi} \int_{0}^{1}\left\{\frac{1}{t-s_{x}+1-i}-\frac{1}{t-s_{x}+1}\right\} d t \\
& -\frac{2}{\pi} \sum_{m=1}^{l(p x) / 2} \int_{0}^{1}\left\{\frac{t-s_{x}+1-i}{4 m^{2}-\left(t-s_{x}+1-i\right)^{2}} \quad-\frac{t-s_{x}+1}{4 m^{2}-\left(t-s_{x}+1\right)^{2}}\right\} d t \\
= & \bar{R} 1\left(s_{x}\right)+O\left(\frac{1}{\log p}\right), \\
L_{2}= & -\frac{i}{\pi} \sum_{m=l(p x)+1}^{(p / 2)-1} \int_{0}^{1} \frac{1}{(2 m-l(p x))+\left(t-s_{x}+1-i\right)} \\
& \times \frac{d t}{(2 m-l(p x))+\left(t-s_{x}+1\right)}=O\left(\frac{1}{\log p}\right)
\end{aligned}
$$

which shows that $\overline{\Delta[p]} \chi_{W_{p}^{\prime}}(x)=\bar{R} 1\left(s_{x}\right)+O(1 / \log p) \quad\left(x \in W_{p} \cap X_{p}\right)$. 
In the same manner, $\overline{\Delta[p]} \chi_{W_{p}}(x)=R 1\left(s_{x}\right)+O(1 / \log p) \quad\left(x \in W_{p}^{\prime} \cap\right.$ $\left.X_{p}\right)$. Thus

$$
\begin{aligned}
d_{2 l}(\Delta[p])= & \int_{0}^{1}(\Delta[p] \overline{\Delta[p]})^{l-1} \Delta[p]\left\{\chi_{W_{p} \cap X_{p}} \bar{R} 1\left(s_{.}\right)+\chi_{W_{p}^{\prime} \cap X_{p}} R 1\left(s_{.}\right)\right\} d x \\
& +O\left(\frac{1}{\log p}\right) \\
= & \int_{0}^{1}(\Delta[p] \overline{\Delta[p]})^{l-1} \Delta[p]\left\{\chi_{W_{p}} \bar{R} 1\left(s_{.}\right)+\chi_{W_{p}^{\prime}} R 1\left(s_{.}\right)\right\} d x \\
& +O\left(\frac{1}{\log p}\right) \\
= & \int_{0}^{1}(\Delta[p] \overline{\Delta[p]})^{l-1}\left\{\chi_{W_{p} \cap X_{p}} \Delta[p]\left(\chi_{W_{p}^{\prime}} R 1\left(s_{.}\right)\right)\right. \\
& +O\left(\frac{1}{\log p}\right) .
\end{aligned}
$$

Since $R 1\left(s_{x}\right)$ is a periodic function with period $1 / p$, we have, in the same manner as above,

$$
\begin{array}{ll}
\Delta[p]\left(\chi_{W_{p}^{\prime}} R 1\left(s_{.}\right)\right)(x)=R^{2} 1\left(s_{x}\right)+O\left(\frac{1}{\log p}\right) & \left(x \in W_{p} \cap X_{p}\right), \\
\Delta[p]\left(\chi_{W_{p}} \bar{R} 1\left(s_{.}\right)\right)(x)=\bar{R}^{2} 1\left(s_{x}\right)+O\left(\frac{1}{\log p}\right) & \left(x \in W_{p}^{\prime} \cap X_{p}\right) .
\end{array}
$$

Repeating this argument, we have

$$
\begin{aligned}
d_{2 l}(\Delta[p]) & =\int_{0}^{1}\left\{\chi_{W_{p}}(x) R^{2 l} 1\left(s_{x}\right)+\chi_{W_{p}^{\prime}}(x) \bar{R}^{2 l} 1\left(s_{x}\right)\right\} d x+O\left(\frac{1}{\log p}\right) \\
& =\frac{1}{2} \int_{0}^{1}\left\{R^{2 l} 1+\bar{R}^{2 l} 1\right\} d s+O\left(\frac{1}{\log p}\right),
\end{aligned}
$$

which gives (16).

We have

$$
\begin{aligned}
R(s, t)= & \frac{1}{\pi} \sum_{m=-\infty}^{\infty}\left\{\frac{1}{2 m+1+t-s+i}-\frac{1}{2 m+1+t-s}\right\} \\
= & -\frac{1}{\pi} \sum_{m=-\infty}^{\infty} \frac{1}{(2 m+1+t-s)\left\{(2 m+1+t-s)^{2}+1\right\}} \\
& -\frac{i}{\pi} \sum_{m=-\infty}^{\infty} \frac{1}{(2 m+1+t-s)^{2}+1} \\
= & -R^{\prime}(s, t)-i R^{\prime \prime}(s, t), \text { say. }
\end{aligned}
$$


Then $R^{\prime}$ is anti-symmetric and $R^{\prime \prime}$ is symmetric, i.e., $R^{\prime \prime}(s, t)=$ $R^{\prime \prime}(t, s)$. Thus

$$
\begin{aligned}
d_{2}(\Delta[\infty]) & =\operatorname{Re} \int_{0}^{1} R^{2} 1 d s \\
& =\operatorname{Re} \int_{0}^{1}\left(-R^{\prime} 1+i R^{\prime \prime} 1\right)\left(R^{\prime} 1+i R^{\prime \prime} 1\right) d s \\
& =-\int_{0}^{1}\left\{\left(R^{\prime} 1\right)^{2}+\left(R^{\prime \prime} 1\right)^{2}\right\} d s \\
& \leq-\frac{1}{\pi^{2}} \int_{0}^{1}\left\{\int_{0}^{1} \frac{d t}{(1+t-s)^{2}+1}\right\}^{2} d s \leq-\frac{1}{25 \pi^{2}} .
\end{aligned}
$$

Thus (15) holds.

LEMMA 7. Let $K$ be an anti-symmetric kernel of type 1, and let $\left(g_{p}\right)_{p=2}^{\infty},\left(h_{p}\right)_{p=2}^{\infty}$ be two sequences in $L^{4}$ such that $\left\|g_{p}\right\|_{L^{4}} \leq 1$, $\left\|h_{p}\right\|_{L^{4}} \leq 1$. Then, for any $l \geq 0$,

$$
\begin{gathered}
\lim _{p \rightarrow \infty}\left\{\int_{0}^{1} K g_{p} \cdot(\Delta[p] \overline{\Delta[p]})^{l} \bar{K} h_{p} d x\right. \\
\left.-d_{2 l}(\Delta[\infty]) \int_{0}^{1} K g_{p} \cdot \bar{K} h_{p} d x\right\}=0 \\
\lim _{p \rightarrow \infty} \int_{0}^{1} K g_{p} \cdot \overline{\Delta[p]}(\Delta[p] \overline{\Delta[p]})^{l} K h_{p} d x=0
\end{gathered}
$$

Equalities (17) and (18) hold with $K g_{p}$ replaced by 1 .

Proof. First we assume that $K$ is of type 0 . Let

$$
\Delta^{\prime}[p](x, y)=\Delta[p](x, y) \chi_{[0, N / p)}(|y-x|) \quad(p \geq 2),
$$

where $N=l(\log p)$. Then $\left\|\Delta[p]-\Delta^{\prime}[p]\right\|_{L^{2}, L^{2}}=O(1 / \log p)$ (cf. Lemma 6), and hence

$$
\begin{aligned}
\int_{0}^{1} K g_{p} \cdot(\Delta[p] \overline{\Delta[p]})^{l} \bar{K} h_{p} d x \\
\quad=\int_{0}^{1} K g_{p} \cdot\left(\Delta^{\prime}[p] \overline{\Delta^{\prime}[p]}\right)^{l} \bar{K} h_{p} d x+O\left(\frac{1}{\log p}\right) .
\end{aligned}
$$

Notice that

$$
\left(\Delta^{\prime}[p] \overline{\Delta^{\prime}[p]}\right)^{l}(x, y)=0 \quad(|y-x|>2 l N / p),
$$


and that $\left(\Delta^{\prime}[p] \overline{\Delta^{\prime}[p]}\right)^{l} 1$ is a periodic function on $[2 l N / p, 1-(2 l N / p))$ with period $2 / p$. Let

$$
\begin{aligned}
\eta_{p}^{(m)}=\frac{p}{2} \int_{2 m / p}^{2(m+1) / p}\left(\Delta^{\prime}[p] \overline{\Delta^{\prime}[p]}\right)^{l} 1 d x-d_{2 l}(\Delta[\infty]) & \\
& (0 \leq m \leq l(p / 2)-1) .
\end{aligned}
$$

Then $\eta_{p}^{(m)}=\eta_{p}^{(l N)}(l N \leq m \leq l(p / 2)-l N-1)$. Lemma 6 shows that

$$
\begin{aligned}
\underset{p \rightarrow \infty}{\limsup }\left|\eta_{p}^{(l N)}\right| & =\limsup _{p \rightarrow \infty}\left|d_{2 l}\left(\Delta^{\prime}[p]\right)-d_{2 l}(\Delta[\infty])\right| \\
& =\limsup _{p \rightarrow \infty}\left|d_{2 l}(\Delta[p])-d_{2 l}(\Delta[\infty])\right|=0 .
\end{aligned}
$$

Since $K$ is of type 0 , we have

$$
\sup \frac{|K h(y)-K h(x)|}{|y-x|} \leq \sup _{s, t \in[0,1)}\left|\frac{\partial}{\partial s} K(s, t)\right|<\infty,
$$

where the supremum in the left-hand side is taken over all $x, y \in$ $[0,1)$ and all $h \in L^{4}$ satisfying $\|h\|_{L^{4}} \leq 1$. Thus

$$
\begin{aligned}
& \int_{0}^{1} K g_{p} \cdot(\Delta[p] \overline{\Delta[p]})^{l} \bar{K} h_{p} d x \\
&=\int_{2 l N / p}^{1-(2 l N / p)} K g_{p}(x) \\
& \times \int_{|y-x| \leq 2 l N / p}\left(\Delta^{\prime}[p] \overline{\Delta^{\prime}[p]}\right)^{l}(x, y) \bar{K} h_{p}(y) d y d x+o(1) \\
&= \int_{2 l N / p}^{1-(2 l N / p)} K g_{p} \cdot \bar{K} h_{p} \cdot\left(\Delta^{\prime}[p] \overline{\Delta^{\prime}[p]}\right)^{l} 1 d x+o(1) \\
&= \frac{2}{p} \sum_{m=l N}^{l(p / 2)-l N-1} K g_{p}\left(\frac{2 m}{p}\right) \bar{K} h_{p}\left(\frac{2 m}{p}\right)\left\{\eta_{p}^{(m)}+d_{2 l}(\Delta[\infty])\right\}+o(1) \\
&= d_{2 l}(\Delta[\infty]) \int_{0}^{1} K g_{p} \cdot \bar{K} h_{p} d x+O\left(\eta_{p}^{(l N)}\right)+o(1) \\
&= d_{2 l}(\Delta[\infty]) \int_{0}^{1} K g_{p} \cdot \bar{K} h_{p} d x+o(1)
\end{aligned}
$$

which shows that (17) holds. Let $K$ be of type 1 . Then there exists a 
sequence $\left(K_{j}\right)_{j=1}^{\infty}$ of kernels of type 0 such that

$$
\left\|K-K_{j}\right\|_{L^{4}, L^{2}} \leq \frac{1}{j} \quad(j \geq 1), \quad \sup _{j \geq 1}\left\|K_{j}\right\|_{L^{4}, L^{4}}<\infty .
$$

Then

$$
\begin{gathered}
\left|\int_{0}^{1} K g_{p} \cdot(\Delta[p] \overline{\Delta[p]}) l \bar{K} h_{p} d x-\int_{0}^{1} K_{j} g_{p} \cdot(\Delta[p] \overline{\Delta[p]})^{l} \bar{K}_{j} h_{p} d x\right| \leq C_{3} / j, \\
\quad\left|\int_{0}^{1} K g_{p} \cdot \bar{K} h_{p} d x-\int_{0}^{1} K_{j} g_{p} \cdot \bar{K}_{j} h_{p} d x\right| \leq C_{3} / j \quad(j \geq 1)
\end{gathered}
$$

for some constant $C_{3}$ independent of $p$ and $j$. Since (17) holds for all $K_{j}(j \geq 1)$, this shows that (17) holds.

Since $\overline{\Delta[p]}(\Delta[p] \overline{\Delta[p]})^{l}$ is anti-symmetric, we have

$$
\int_{0}^{1} \overline{\Delta[p]}(\Delta[p] \overline{\Delta[p]})^{l} 1 d x=0 .
$$

Hence, in the same manner as above, we obtain (18). Analogously, we can replace $K g_{p}$ by 1 .

LEMMA 8. Let $K$ be an anti-symmetric kernel of type 1. Then, for any $l \geq 0$,

$$
\lim _{p \rightarrow \infty} d_{2 l}(\Delta[p]+K) \quad\left(=d_{2 l}(\Delta[\infty]+K), \text { say }\right)
$$

exists, and we can write

$$
d_{2 l}(\Delta[\infty]+K)=\sum_{k=0}^{l} c_{2 k}^{(2 l)} d_{2 l-2 k}(K)
$$

so that $c_{2 k}^{(m)}(0 \leq k \leq l(m / 2), m \geq 0)$ satisfy

$$
c_{0}^{(m)}=1, \quad c_{2 k}^{(2 k)}=d_{2 k}(\Delta[\infty]) \quad(m \geq 0, k \geq 0),
$$

(21) $c_{2 k}^{(m)}=\sum_{j=0}^{k} c_{2 k-2 j}^{(m-2 j-1)} d_{2 j}(\Delta[\infty])$

$$
\left(0 \leq k \leq l\left(\frac{m-1}{2}\right), m \geq 0\right)
$$


Proof. We say that a $2 l$-tuple $\left(\tau_{1}, \ldots, \tau_{2 l}\right), \tau_{j}= \pm 1$ is negligible if there exist two integers $j_{0}, j_{0}^{\prime}\left(1 \leq j_{0}<j_{0}^{\prime} \leq 2 l\right)$ such that $j_{0}^{\prime}-j_{0}-1$ is odd, $\tau_{j}=-1 \quad\left(j_{0} \leq j \leq j_{0}^{\prime}\right)$ and $\tau_{j_{0}-1}=\tau_{j_{0}^{\prime}+1}=1$. (We put $\tau_{0}=\tau_{2 l+1}=1$. Hence $\tau_{j_{0}-1}=1$ if $j_{0}=1$, and $\tau_{j_{0}^{\prime}+1}=1$ if $j_{0}^{\prime}=2 l$.) Let $\tau(\Delta[p])=-1(p \geq 2), \tau(K)=1$. Lemmas 6 and 7 show that $d_{2 l}(\Delta[\infty]+K)$ exists and

$$
\begin{aligned}
d_{2 l}(\Delta[\infty]+K) & =\lim _{p \rightarrow \infty} \sum_{\left(K_{1}, \ldots, K_{2 l}\right), K_{j}=\Delta[p], K} \int_{0}^{1} K_{1} \bar{K}_{2} \cdots K_{2 l-1} \bar{K}_{2 l} 1 d x \\
& =\lim _{p \rightarrow \infty} \sum_{(p)} \int_{0}^{1} K_{1} \bar{K}_{2} \cdots K_{2 l-1} \bar{K}_{2 l} 1 d x
\end{aligned}
$$

where $\sum_{(p)}$ is the summation over all $2 l$-tuples $\left(K_{1}, \ldots, K_{2 l}\right), K_{j}=$ $\Delta[p], K$ such that $\left(\tau\left(K_{1}\right), \ldots, \tau\left(K_{2 l}\right)\right)$ is not negligible. If $\left(\tau\left(K_{1}\right), \ldots\right.$, $\left.\tau\left(K_{2 l}\right)\right)$ is not negligible, then $K$ appears even times in $\left(K_{1}, \ldots, K_{2 l}\right)$. We can choose $j_{1}<j_{2}<\cdots<j_{2 \nu}$ so that $K_{j_{\mu}}=K \quad(1 \leq \mu \leq 2 \nu)$, $K_{j}=\Delta[p]\left(j \notin\left\{j_{\mu}\right\}_{\mu=1}^{2 \nu}\right)$. Then $j_{1}-1, j_{\mu+1}-j_{\mu}-1(1 \leq \mu \leq 2 \nu-1)$, $2 l-j_{2 \nu}$ are even. Notice that

$$
d_{2 j}(K)=\int_{0}^{1}(\bar{K} K)^{j} 1 d x \quad(j \geq 0)
$$

Thus we can write

$$
d_{2 l}(\Delta[\infty]+K)=\sum_{k=0}^{l} c_{2 k}^{(2 l)} d_{2 l-2 k}(K)
$$

Let $\kappa_{0}$ be an operator defined by $h \in L^{2} \rightarrow\left(\int_{0}^{1} h d x\right) \chi_{[0,1)}$. We put $Y_{p,-1}(t)=1$,

$$
Y_{p, m}(t)= \begin{cases}\int_{0}^{1} K_{p, t}^{m / 2} 1 d x & (m \text { is even }) \\ \int_{0}^{1}\left(\kappa_{0}+t \overline{\Delta[p]}\right) K_{p, t}^{(m-1) / 2} 1 d x & (m \text { is odd })\end{cases}
$$

where $K_{p, t}=\left(\kappa_{0}+t \Delta[p]\right)\left(\kappa_{0}+t \overline{\Delta[p]}\right)$. Then $Y_{\infty, m}(t)=\lim _{p \rightarrow \infty} Y_{p, m}(t)$ exists, and $c_{2 k}^{(2 l)}$ equals the $t^{2 k}$-coefficient of $Y_{\infty, 2 l}(t)$. Evidently, (20) 
holds. Since $\int_{0}^{1} \Delta[p](\overline{\Delta[p]} \Delta[p])^{j} 1 d x=0 \quad(j \geq 0)$, we have inductively

$$
\begin{aligned}
Y_{p, 2 l}(t)= & Y_{p, 2 l-1}(t)+t \int_{0}^{1} \Delta[p]\left(\kappa_{0}+t \overline{\Delta[p]}\right) K_{p, t}^{l-1} 1 d x \\
= & Y_{p, 2 l-1}(t)+t^{2} \int_{0}^{1} \Delta[p] \overline{\Delta[p]} K_{p, t}^{l-1} 1 d x \\
= & Y_{p, 2 l-1}(t)+t^{2} d_{2}(\Delta[p]) Y_{p, 2 l-3}(t) \\
& +t^{3} \int_{0}^{1} \Delta[p] \overline{\Delta[p]} \Delta[p]\left(\kappa_{0}+t \overline{\Delta[p]}\right) K_{p, t}^{l-2} 1 d x \\
= & Y_{p, 2 l-1}(t)+t^{2} d_{2}(\Delta[p]) Y_{p, 2 l-3}(t) \\
& +t^{4} \int_{0}^{1}(\Delta[p] \overline{\Delta[p]})^{2} K_{p, t}^{l-2} 1 d x \\
= & \cdots=\sum_{j=0}^{l} t^{2 j} d_{2 j}(\Delta[p]) Y_{p, 2 l-2 j-1}(t) .
\end{aligned}
$$

Letting $p$ tend to infinity, we have

$$
Y_{\infty, 2 l}(t)=\sum_{j=0}^{l} t^{2 j} d_{2 \jmath}(\Delta[\infty]) Y_{\infty, 2 l-2 j-1}(t) .
$$

In the same manner,

$$
Y_{\infty, 2 l+1}(t)=\sum_{j=0}^{l} t^{2 j} d_{2 j}(\Delta[\infty]) Y_{\infty, 2 l-2 j}(t) .
$$

Thus

$$
Y_{\infty, m}(t)=\sum_{j=0}^{l(m / 2)} t^{2 \jmath} d_{2 j}(\Delta[\infty]) Y_{\infty, m-2 \jmath-1}(t) .
$$

Comparing the $t^{2 k}$-coefficients of both sides, we obtain (21).

LEMMA 9. Let $K$ be an anti-symmetric kernel of type 1. Then, for any $0<\delta \leq 1$,

$$
\lim _{p \rightarrow \infty} \gamma^{*}(\delta \Delta[p]+\delta K) \quad\left(=\gamma^{*}(\delta \Delta[\infty]+\delta K), \text { say }\right)
$$

exists; we write $\gamma^{*}(\delta \Delta[\infty])$ if $K=0$. Moreover,

$$
\gamma^{*}(\delta \Delta[\infty]+\delta K)=\gamma^{*}(\delta \Delta[\infty]) \gamma^{*}\left(\gamma^{*}(\delta \Delta[\infty]) \delta K\right) .
$$

Proof. First we show that $\gamma^{*}(\delta \Delta[\infty]+\delta K)$ and $\gamma^{*}(\delta \Delta[\infty])$ exist. Define $\gamma^{*}(w ; \Delta[p]+K), T(w ; \Delta[p]+K)(w \in U)$ for $\varepsilon_{0}=$ 
$\left(12+3\|K\|_{L^{2}, L^{2}}\right)^{-1}$ in the same manner as in Lemma 5; we have $\varepsilon_{0} \leq\left(3\|\Delta[p]+K\|_{L^{2}, L^{2}}\right)^{-1}$ because $\|\Delta[p]\|_{L^{2}, L^{2}} \leq 4$. Lemma 8 shows that

$$
\begin{aligned}
\lim _{p \rightarrow \infty} & w^{2} \varepsilon_{0}^{2} \int_{0}^{1}(\Delta[p]+K) T(w ; \Delta[p]+K)^{m+1}(\overline{\Delta[p]+K}) 1 d x \\
= & \sum_{l=0}^{\infty} w^{2 l+2} \varepsilon_{0}^{2 l+2} \frac{(l+1) \cdots(l+m)}{m !} d_{2 l+2}(\Delta[\infty]+K) \quad(m \geq 0) .
\end{aligned}
$$

Since (14) holds with $K$ replaced by any $\Delta[p]+K(p \geq 2),(13)$ exists with $K$ replaced by $\Delta[\infty]+K$, i.e.,

$$
\lim _{p \rightarrow \infty} \gamma^{*}(w ; \Delta[p]+K) \quad\left(=\gamma^{*}(w ; \Delta[\infty]+K), \text { say }\right)
$$

exists. Since

$$
\gamma^{*}(\delta ; \Delta[p]+K)=\gamma^{*}(\delta \Delta[p]+\delta K) \quad(p \geq 2),
$$

$\gamma^{*}(\delta \Delta[\infty]+\delta K) \quad\left(=\gamma^{*}(\delta ; \Delta[\infty]+K)\right)$ exists. Putting $K=0$, we see that $\gamma^{*}(\delta \Delta[\infty])$ exists.

Next we show that $\gamma^{*}(w ; \Delta[\infty]+K)$ and $\gamma^{*}\left(\gamma^{*}(w ; \Delta[\infty]) w ; K\right)$ are analytic in a domain containing $(0,1]$. The convergence of $(23)$ is uniform in $U$. By Lemma $5, \gamma^{*}(w ; \Delta[p]+K)$ is analytic in $U$, and hence $\gamma^{*}(w ; \Delta[\infty]+K)$ is analytic in $U$. The definition of $\gamma^{*}(\cdot)$ immediately shows that

$$
\gamma^{*}(\operatorname{Re} w ; \Delta[p])=\gamma^{*}(\operatorname{Re} w \Delta[p]) \leq 1 \quad(w \in U) .
$$

Letting $p$ tend to infinity, we have $\gamma^{*}(\operatorname{Re} w ; \Delta[\infty]) \leq 1 \quad(w \in U)$. Since $\gamma^{*}(w ; \Delta[\infty])$ is analytic in $U$, there exists $0<\eta<\pi / 8$ such that

$$
\left|\gamma^{*}(w ; \Delta[\infty])\right| \leq \frac{4}{3}, \quad\left|\arg \gamma^{*}(w ; \Delta[\infty])\right| \leq \frac{\pi}{8}
$$

in $U_{\eta}=\{w \in \mathbb{C} ;|w|<4 / 3,|\arg w|<\eta\}$. Then $\gamma^{*}(w ; \Delta[\infty]) w \in U$ $\left(w \in U_{\eta}\right)$. Thus, by Lemma $5, \gamma^{*}\left(\gamma^{*}(w ; \Delta[\infty]) w ; K\right)$ is analytic in $U_{\eta}$.

By the theorem of identity, it is sufficient to show that (22) holds for $0<\delta<\left(8+2\|K\|_{L^{2}, L^{2}}\right)^{-1}$. Since

$$
\lim _{l \rightarrow \infty} d_{2 l}(\delta \Delta[p])=\lim _{l \rightarrow \infty} d_{2 l}(\delta \Delta[p]+\delta K)=0,
$$

(12) holds for $\delta \Delta[p], \delta \Delta[p]+\delta K(p \geq 2)$. Letting $p$ tend to infinity, we have

$$
\gamma^{*}(\delta \Delta[\infty])=\sum_{l=0}^{\infty} d_{2 l}(\delta \Delta[\infty])=\sum_{l=0}^{\infty} \delta^{2 l} d_{2 l}(\Delta[\infty])
$$




$$
\gamma^{*}(\delta \Delta[\infty]+\delta K)=\sum_{l=0}^{\infty} \delta^{2 l} d_{2 l}(\Delta[\infty]+K)
$$

Let

$$
\mu_{m}=\sum_{k=0}^{\infty} \delta^{2 k} c_{2 k}^{(m+2 k)} \quad(m \geq 0),
$$

where $c_{2 k}^{(m)}(0 \leq k \leq l(m / 2), m \geq 0)$ are numbers in Lemma 8. Then

$$
\mu_{0}=\sum_{k=0}^{\infty} \delta^{2 k} c_{2 k}^{(2 k)}=\gamma^{*}(\delta \Delta[\infty]),
$$

by (20). Equality (21) yields that

$$
\begin{aligned}
\mu_{m} & =\sum_{k=0}^{\infty} \delta^{2 k} \sum_{j=0}^{k} c_{2 k-2 j}^{(m+2 k-2 j-1)} d_{2 j}(\Delta[\infty]) \\
& =\sum_{j=0}^{\infty} \delta^{2 j} d_{2 j}(\Delta[\infty]) \sum_{k=j}^{\infty} \delta^{2(k-j)} c_{2(k-j)}^{(m-1+2(k-j))} \\
& =\mu_{m-1} \mu_{0} \quad(m \geq 1),
\end{aligned}
$$

which gives

$$
\mu_{m}=\mu_{0}^{m+1}=\gamma^{*}(\delta \Delta[\infty])^{m+1} \quad(m \geq 1) .
$$

Thus, by (21),

$$
\begin{aligned}
\gamma^{*}(\delta & {[\infty]+\delta K)=\sum_{l=0}^{\infty} \delta^{2 l} d_{2 l}(\Delta[\infty]+K) } \\
& =\sum_{l=0}^{\infty} \delta^{2 l} \sum_{k=0}^{l} c_{2 k}^{(2 l)} d_{2 l-2 k}(K)=\sum_{k=0}^{\infty} \sum_{l=k}^{\infty} \delta^{2 l} c_{2 k}^{(2 l)} d_{2 l-2 k}(K) \\
& =\sum_{k=0}^{\infty} \sum_{j=0}^{\infty} \delta^{2 j+2 k} c_{2 k}^{(2 j+2 k)} d_{2 j}(K)=\sum_{j=0}^{\infty} \mu_{2 j} \delta^{2 j} d_{2 j}(K) \\
& =\sum_{j=0}^{\infty} \gamma^{*}(\delta \Delta[\infty])^{2 j+1} \delta^{2 j} d_{2 j}(K)=\gamma^{*}(\delta \Delta[\infty]) \gamma^{*}\left(\gamma^{*}(\delta \Delta[\infty]) \delta K\right)
\end{aligned}
$$

LEMma 10. We inductively define a sequence $\left(\gamma_{n}^{*}\right)_{n=1}^{\infty}$ of positive numbers by

$$
\gamma_{1}^{*}=\gamma^{*}(\Delta[\infty]), \quad \gamma_{n}^{*}=\gamma_{n-1}^{*} \gamma^{*}\left(\gamma_{n-1}^{*} \Delta[\infty]\right) \quad(n \geq 2) .
$$


Then

$$
\lim _{p_{1} \rightarrow \infty} \lim _{p_{2} \rightarrow \infty} \cdots \lim _{p_{n} \rightarrow \infty} \gamma^{*}\left(H\left[p_{1}, \ldots, p_{n}\right]-H\right)=\gamma_{n}^{*} \quad(n \geq 1),
$$

where $\lim _{p_{n} \rightarrow \infty}$ is taken first and $\lim _{p_{1} \rightarrow \infty}$ is taken last.

Proof. We define a sequence $\left(\lambda_{n}\right)_{n=1}^{\infty}$ of positive numbers by $\lambda_{1}=$ $\gamma^{*}(\Delta[\infty]), \lambda_{n}=\gamma^{*}\left(\lambda_{1} \ldots \lambda_{n-1} \Delta[\infty]\right)(n \geq 2)$. Then $\gamma_{n}^{*}=\lambda_{1} \cdots \lambda_{n}$ $(n \geq 1)$. Fixing an $(n-1)$-tuple $\left(p_{1}, \ldots, p_{n-1}\right)(n \geq 2)$ of integers larger than or equal to 3 , we study

$$
\begin{aligned}
\lim _{p_{n} \rightarrow \infty} & \gamma^{*}\left(H\left[p_{1}, \ldots, p_{n}\right]-H\right) \\
= & \lim _{p_{n} \rightarrow \infty} \gamma^{*}\left(\Delta\left[p_{1}, \ldots, p_{n}\right]+\left(H\left[p_{1}, \ldots, p_{n-1}\right]-H\right)\right) .
\end{aligned}
$$

Put $I_{0}=\left[0,1 /\left(p_{1} \cdots p_{n-1}\right)\right), I_{j}=\left(I_{0}+j /\left(p_{1} \cdots p_{n-1}\right)\right) \quad(0 \leq j \leq$ $\left.\left(p_{1} \ldots p_{n-1}\right)-1\right)$. Then

$$
\begin{gathered}
\left(H\left[p_{1}, \ldots, p_{n-1}\right]-H\right)(x, y)=0 \quad\left(x, y \in I_{j}\right), \\
\left|\left(H\left[p_{1}, \ldots, p_{n-1}\right]-H\right)(x, y)\right| \\
\leq \frac{2}{\pi} p_{1} \cdots p_{n-1}+\frac{1}{\pi} \frac{1}{|y-x|} \quad\left(x \in I_{j}, y \in I_{k}, j \neq k\right),
\end{gathered}
$$

which shows that $H\left[p_{1}, \ldots, p_{n-1}\right]-H$ is of type 1 . Let

$$
\begin{gathered}
\Delta^{\prime}\left[p_{1}, \ldots, p_{n}\right](x, y)=\Delta\left[p_{1}, \ldots, p_{n}\right](x, y) \chi_{\left[0, \frac{N^{\prime}}{p_{1} \ldots p_{n}}\right)}(|y-x|), \\
\Delta^{\prime}\left[\prod_{j=1}^{n} p_{j}\right](x, y)=\Delta\left[\prod_{j=1}^{n} p_{j}\right](x, y) \chi_{\left[0, \frac{N^{\prime}}{p_{1} \ldots p_{n}}\right)}(|y-x|)
\end{gathered}
$$

$\left(N^{\prime}=\imath\left(\log \left(p_{1} \ldots p_{n}\right)\right)\right.$. Then

$$
\begin{gathered}
\lim _{p_{n} \rightarrow \infty}\left\|\Delta\left[p_{1}, \ldots, p_{n}\right]-\Delta^{\prime}\left[p_{1}, \ldots, p_{n}\right]\right\|_{L^{4}, L^{4}}=0, \\
\lim _{p_{n} \rightarrow \infty}\left\|\Delta\left[\prod_{j=1}^{n} p_{j}\right]-\Delta^{\prime}\left[\prod_{j=1}^{n} p_{j}\right]\right\|_{L^{4}, L^{4}}=0
\end{gathered}
$$

(cf. Lemmas 6 and 7). Since

$$
\begin{aligned}
\Delta^{\prime}\left[p_{1}, \ldots, p_{n}\right](x, y)=\Delta^{\prime}\left[\prod_{j=1}^{n} p_{j}\right] & (x, y) \\
& \left(x, y \in I_{j}, 0 \leq j \leq\left(p_{1} \cdots p_{n-1}\right)-1\right),
\end{aligned}
$$


we have

$$
\begin{gathered}
\lim _{p_{n} \rightarrow \infty}\left\|\Delta\left[p_{1}, \ldots, p_{n}\right]-\Delta\left[\prod_{j=1}^{n} p_{j}\right]\right\|_{L^{4}, L^{2}} \\
=\lim _{p_{n} \rightarrow \infty}\left\|\Delta^{\prime}\left[p_{1}, \ldots, p_{n}\right]-\Delta^{\prime}\left[\prod_{j=1}^{n} p_{j}\right]\right\|_{L^{4}, L^{2}}=0,
\end{gathered}
$$

and hence, in the same manner as in the proof of the existence of (23),

$$
\begin{aligned}
\lim _{p_{n} \rightarrow \infty} \gamma^{*}\left(\Delta\left[p_{1}, \ldots, p_{n}\right]+\left(H\left[p_{1}, \ldots, p_{n-1}\right]-H\right)\right) \\
\quad=\lim _{p_{n} \rightarrow \infty} \gamma^{*}\left(\Delta\left[\prod_{j=1}^{n} p_{j}\right]+\left(H\left[p_{1}, \ldots, p_{n-1}\right]-H\right)\right) .
\end{aligned}
$$

Using (22) with $\delta=1, K=H\left[p_{1}, \ldots, p_{n-1}\right]-H$, we have

$$
\begin{aligned}
& \lim _{p_{n} \rightarrow \infty} \gamma^{*}\left(H\left[p_{1}, \ldots, p_{n}\right]-H\right)=\gamma^{*}\left(\Delta[\infty]+\left(H\left[p_{1}, \ldots, p_{n-1}\right]-H\right)\right) \\
& =\lambda_{1} \gamma^{*}\left(\lambda_{1}\left(H\left[p_{1}, \ldots, p_{n-1}\right]-H\right)\right) .
\end{aligned}
$$

In the same manner, using (22) with $\delta=\lambda_{1}, K=H\left[p_{1}, \ldots, p_{n-2}\right]-$ $H$, we have

$$
\begin{aligned}
& \lim _{p_{n-1} \rightarrow \infty} \lim _{p_{n} \rightarrow \infty} \gamma^{*}\left(H\left[p_{1}, \ldots, p_{n}\right]-H\right) \\
& \quad=\lambda_{1} \gamma^{*}\left(\lambda_{1} \Delta[\infty]\right) \gamma^{*}\left(\gamma^{*}\left(\lambda_{1} \Delta[\infty]\right) \lambda_{1}\left(H\left[p_{1}, \ldots, p_{n-2}\right]-H\right)\right) \\
& \quad=\lambda_{1} \lambda_{2} \gamma^{*}\left(\lambda_{1} \lambda_{2}\left(H\left[p_{1}, \ldots, p_{n-2}\right]-H\right)\right) .
\end{aligned}
$$

Repeating this argument,

$$
\begin{aligned}
\lim _{p_{1} \rightarrow \infty} & \cdots \lim _{p_{n} \rightarrow \infty} \gamma^{*}\left(H\left[p_{1}, \ldots, p_{n}\right]-H\right) \\
& =\lambda_{1} \cdots \lambda_{n-1} \lim _{p_{1} \rightarrow \infty} \gamma^{*}\left(\lambda_{1} \cdots \lambda_{n-1} \Delta\left[p_{1}\right]\right)=\lambda_{1} \ldots \lambda_{n}=\gamma_{n}^{*} .
\end{aligned}
$$

This completes the proof of our lemma.

We now give the proof of our theorem. By Proposition 2, there exists a positive integer $n_{0}$ such that

$$
\sup \gamma\left(\Gamma\left(p_{1}, \ldots, p_{n}\right)\right) \leq 10^{-5} \quad\left(n \geq n_{0}\right),
$$

where the supremum is taken over all $n$-tuples $\left(p_{1}, \ldots, p_{n}\right)$ of integers larger than or equal to 3 . By Lemma 10 , we can inductively choose a sequence $\left(p_{n}^{0}\right)_{n=1}^{\infty}$ of integers larger than or equal to 3 so that

$$
\frac{1}{2} \gamma_{n}^{*} \leq \gamma^{*}\left(H\left[p_{1}^{0}, \ldots, p_{n}^{0}\right]-H\right) \leq 2 \gamma_{n}^{*} \quad(n \geq 1),
$$


where $\left(\gamma_{n}^{*}\right)_{n=1}^{\infty}$ is the sequence in Lemma 10. We show that $\Gamma_{n}=$ $\Gamma\left(p_{1}^{0}, \ldots, p_{n}^{0}\right) \quad(n \geq 1)$ are required cranks. We may assume that $n \geq n_{0}$. Lemma 3 shows that

$$
\begin{aligned}
\frac{1}{4} \gamma^{*}\left(H\left[p_{1}^{0}, \ldots, p_{n}^{0}\right]-H\right) & \leq \gamma^{*}\left(H\left[p_{1}^{0}, \ldots, p_{n}^{0}\right]\right) \\
& \leq 4 \gamma^{*}\left(H\left[p_{1}^{0}, \ldots, p_{n}^{0}\right]-H\right),
\end{aligned}
$$

and hence

$$
\frac{1}{8} \gamma_{n}^{*} \leq \gamma^{*}\left(H\left[p_{1}^{0}, \ldots, p_{n}^{0}\right]\right) \leq 8 \gamma_{n}^{*}
$$

Thus, by (11),

$$
\frac{1}{8 \pi} \gamma_{n}^{*} \leq \gamma\left(\Gamma_{n}\right) \leq \frac{8}{\pi} \gamma_{n}^{*}
$$

Using (24) and (25), we have $\gamma_{n}^{*} \leq 8 \pi \cdot 10^{-5}$. Recall (15), and notice that $d_{2 l}(\Delta[\infty]) \leq 4^{l} \quad(l \geq 1)$. Since $\lim _{l \rightarrow \infty} d_{2 l}\left(\gamma_{n}^{*} \Delta[p]\right)=0$, (12) holds for $\gamma_{n}^{*} \Delta[p]$. Letting $p$ tend to infinity, we have

$$
\begin{aligned}
\gamma_{n+1}^{*} & =\gamma_{n}^{*} \gamma^{*}\left(\gamma_{n}^{*} \Delta[\infty]\right)=\gamma_{n}^{*} \sum_{l=0}^{\infty} d_{2 l}\left(\gamma_{n}^{*} \Delta[\infty]\right) \\
& =\gamma_{n}^{*} \sum_{l=0}^{\infty} \gamma_{n}^{*^{2 l}} d_{2 l}(\Delta[\infty]) \leq \gamma_{n}^{*}-\frac{1}{25 \pi^{2}} \gamma_{n}^{*^{3}}+\sum_{l=2}^{\infty} 4^{l} \gamma_{n}^{*^{2 l+1}} \\
& \leq \gamma_{n}^{*}-10^{-3} \gamma_{n}^{*^{3}}, \\
& \gamma_{n+1}^{*} \geq \gamma_{n}^{*}-\sum_{l=1}^{\infty} 4^{l} \gamma_{n}^{*^{2 l+1}} \geq \gamma_{n}^{*}-10 \gamma_{n}^{*^{3}}, \quad \text { i.e. } \\
& \gamma_{n}^{*}-10 \gamma_{n}^{*^{3}} \leq \gamma_{n+1}^{*} \leq \gamma_{n}^{*}-10^{-3} \gamma_{n}^{*^{3}} .
\end{aligned}
$$

Since this holds for all $n \geq n_{0}$, a simple induction yields that

$$
\frac{1}{C_{4}} \frac{1}{\sqrt{n}} \leq \gamma_{n}^{*} \leq C_{4} \frac{1}{\sqrt{n}} \quad\left(n \geq n_{0}\right)
$$

for some absolute constant $C_{4}$. Using (25) again,

$$
\frac{1}{8 \pi C_{4}} \frac{1}{\sqrt{n}} \leq \gamma\left(\Gamma_{n}\right) \leq \frac{8}{\pi} C_{4} \frac{1}{\sqrt{n}} \quad\left(n \geq n_{0}\right) .
$$

This completes the proof of our theorem.

REMARK 11. It is not known whether $\gamma(\cdot)$ is semi-additive [4, p. 11]. For $0<\eta \leq 1$, we define $B_{p}^{\eta}(x)$ replacing $1 / 2 p$ by $\eta / 2 p$ in the definition of $B_{p}(x)$. Then cranks $\Gamma^{\eta}\left(p_{1}, \ldots, p_{n}\right)$ of degree $n$ are 
analogously defined. We see that there exists a crank $\Gamma_{n}^{\eta}$ of degree $n$ such that $\gamma\left(\Gamma_{n}^{\eta}\right) \leq C_{\eta} / \sqrt{n}$, where $C_{\eta}$ is a constant depending only on $\eta$. Adding some segments (perpendicular to the $x$-axis) to $\Gamma_{n}^{\eta}$, we obtain an arc $\widetilde{\Gamma}_{n}^{\eta}$ connecting 0 and 1 . Then the diameter of $\widetilde{\Gamma}_{n}^{\eta}$ is larger than or equal to 1 . Since $\widetilde{\Gamma}_{n}^{\eta}$ is connected, $\gamma\left(\widetilde{\Gamma}_{n}^{\eta}\right) \geq 1 / 4[\mathbf{4}, \mathbf{p}$. 9]. Hence, from the point of view of the above semi-additive problem, it seems interesting to compute $\gamma\left(\widetilde{\Gamma}_{n}^{\eta}-\Gamma_{n}^{\eta}\right)$.

4. Another application of Proposition 1. In this section, we show another application of our method. Let $E$ be a compact set on $\mathbb{R}$. Pommerenke [11] showed that

$$
\gamma(E)=|E| / 4,
$$

$$
f_{E}(z)=\left\{1-\exp \left(\frac{1}{2} \int_{E} \frac{d t}{t-z}\right)\right\} /\left\{1+\exp \left(\frac{1}{2} \int_{E} \frac{d t}{t-z}\right)\right\}
$$

We deduce (26), (27) from (3), (10); our method explains a quarter and $(27)$. Let $L^{2}(\mathbb{R})$ denote the $L^{2}$ space of functions on $\mathbb{R}$, and let $M_{E}$ denote the multiplier: $h \in L^{2}(\mathbb{R}) \rightarrow \chi h \in L^{2}(\mathbb{R})$, where $\chi=\chi_{E}$. We inductively define a sequence $\left(H_{E}^{(m)}\right)_{m=0}^{\infty}$ of operators from $L^{2}(\mathbb{R})$ to itself by $H_{E}^{(0)}=M_{E}, H_{E}^{(m)}=H M_{E} H_{E}^{(m-1)}(m \geq 1)$. Notice that

$$
\begin{aligned}
\gamma(E) & =\frac{1}{\pi} \gamma^{*}\left(M_{E} H M_{E}\right), \\
d_{2 l}\left(M_{E} H M_{E}\right) & =\int_{E} H_{E}^{(2 l)} \chi d x \quad\left(l \geq 0, \chi=\chi_{E}\right) .
\end{aligned}
$$

We also remark that

$$
H(g \cdot H h)+H(H g \cdot h)=H g \cdot H h-g h \quad\left(g, h \in L^{2}(\mathbb{R})\right) .
$$

We first show that, for any $m \geq 1$,

$$
\chi H \chi \cdot H_{E}^{(m)} \chi=(m+1) \chi H_{E}^{(m+1)} \chi+m \chi H_{E}^{(m-1)} \chi .
$$

Equality $(28)$ shows that $2 H(\chi H \chi)=(H \chi)^{2}-\chi \chi$, which gives $\chi H \chi$. $H_{E}^{(1)} \chi=2 \chi H_{E}^{(2)} \chi+\chi H_{E}^{(0)} \chi$. Suppose that (29) holds for $m$. Using (28) with $g=\chi, h=\chi H_{E}^{(m)} \chi$, we have

$$
\begin{aligned}
\chi H \chi & \cdot H_{E}^{(m+1)} \chi=\chi H \chi \cdot H\left(\chi H_{E}^{(m)} \chi\right) \\
& =\chi H\left\{\chi H\left(\chi H_{E}^{(m)} \chi\right)+H \chi \cdot \chi H_{E}^{(m)} \chi\right\}+\chi\left\{\chi \cdot \chi H_{E}^{(m)} \chi\right\} \\
& =\chi H_{E}^{(m+2)} \chi+\chi H\left\{(m+1) \chi H_{E}^{(m+1)} \chi+m \chi H_{E}^{(m-1)} \chi\right\}+\chi H_{E}^{(m)} \chi \\
& =(m+2) \chi H_{E}^{(m+2)} \chi+(m+1) \chi H_{E}^{(m)} \chi, \quad \text { i.e., }
\end{aligned}
$$


(29) holds for $m+1$. Thus (29) holds for all $m \geq 1$.

We next show that

$$
\int_{E} H_{E}^{(2 l)} \chi d x=\frac{(-1)^{l}}{2 l+1}|E| \quad(l \geq 0) .
$$

We put $a_{2 l}=\int_{E} H_{E}^{(2 l)} \chi d x \quad(l \geq 0)$. Evidently, $a_{0}=|E|$. Suppose that $a_{2 l-2}=\left\{(-1)^{l-1} /(2 l-1)\right\}|E|$. Equality $(29)(m=2 l-1)$ shows that

$$
\begin{aligned}
\int_{E} H \chi \cdot H_{E}^{(2 l-1)} \chi d x & =2 l \int_{E} H_{E}^{(2 l)} \chi d x+(2 l-1) \int_{E} H_{E}^{(2 l-2)} \chi d x \\
& =2 l a_{2 l}+(2 l-1) a_{2 l-2} .
\end{aligned}
$$

Since the adjoint operator of $H$ equals $-H$, we have

$$
\int_{E} H \chi \cdot H_{E}^{(2 l-1)} \chi d x=-\int_{E} H\left\{\chi H_{E}^{(2 l-1)} \chi\right\} d x=-a_{2 l} \text {. }
$$

Thus $-a_{2 l}=2 l a_{2 l}+(2 l-1) a_{2 l-2}$, which yields that

$$
a_{2 l}=-\frac{2 l-1}{2 l+1} a_{2 l-2}=\frac{(-1)^{l}}{2 l+1}|E| \text {. }
$$

Now the deduction of $(26)$ is immediate. By (30),

$$
\lim _{l \rightarrow \infty} d_{2 l}\left(M_{E} H M_{E}\right)=\lim _{l \rightarrow \infty} \int_{E} H_{E}^{(2 l)} \chi d x=0 .
$$

Hence we can apply (3). Leibniz's formula and (30) yield that

$$
\begin{aligned}
\gamma(E) & =\frac{1}{\pi} \gamma^{*}\left(M_{E} H M_{E}\right)=\frac{1}{\pi} \sum_{l=0}^{\infty} d_{2 l}\left(M_{E} H M_{E}\right) \\
& =\frac{1}{\pi} \sum_{l=0}^{\infty} \frac{(-1)^{l}}{2 l+1}|E|=\frac{1}{4}|E| .
\end{aligned}
$$

Last, we deduce (27) from (10). Equality (10) gives that

$$
f_{E}(z)=-\frac{1}{\pi}\left\{\int_{E} \frac{d s}{s-z}+\int_{E} \frac{H_{E}^{(1)} h_{E}(s)}{s-z} d s\right\} /\left\{1+\frac{1}{\pi} \int_{E} \frac{h_{E}(s)}{s-z} d s\right\},
$$

where $h_{E}(s)$ is the function which attains $\gamma^{*}\left(M_{E} H M_{E}\right)$. We show that this equals the function in the right-hand side of (27). Let

$$
\begin{aligned}
& u_{0}(z)=1, \quad u_{m}(z)=\frac{1}{\pi} \int_{E} \frac{H_{E}^{(m-1)} \chi(s)}{s-z} d s, \\
& v_{m}(z)=\frac{1}{\pi} \int_{E} \frac{H \chi(s) H_{E}^{(m-2)} \chi(s)}{s-z} d s \quad(m \geq 1),
\end{aligned}
$$


where $H_{E}^{(-1)} \chi=\chi$. Let

$$
P_{t}(z)=\sum_{m=0}^{\infty} t^{m} u_{m}(z) \quad(t \in \mathbb{C},|t|<1) .
$$

We begin by showing that

$$
\left(1+t^{2}\right) \frac{\partial}{\partial t} P_{t}(z)=u_{1}(z) P_{t}(z) \quad(0<t<1) .
$$

Let $m \geq 1$. We have, on $\mathbb{R}$,

$$
\begin{aligned}
& \lim _{\eta \downarrow 0}\left\{u_{m+1}(\cdot+i \eta)+v_{m+1}(\cdot+i \eta)\right\} \\
&= H\left(\chi H_{E}^{(m)} \chi\right)+i \chi H_{E}^{(m)} \chi \\
&+H\left\{\chi H \chi \cdot H_{E}^{(m-1)} \chi\right\}+i \chi H \chi \cdot H_{E}^{(m-1)} \chi \\
&= H\left\{\chi H\left(\chi H_{E}^{(m-1)} \chi\right)+H \chi \cdot \chi H_{E}^{(m-1)} \chi\right\} \\
&+i\left\{\chi H\left(\chi H_{E}^{(m-1)} \chi\right)+H \chi \cdot \chi H_{E}^{(m-1)} \chi\right\}, \\
& \lim _{\eta \downarrow 0} u_{1}(\cdot+i \eta) u_{m}(\cdot+i \eta) \\
&=\{H \chi+i \chi\}\left\{H\left(\chi H_{E}^{(m-1)} \chi\right)+i \chi H_{E}^{(m-1)} \chi\right\} \\
&= H \chi \cdot H\left(\chi H_{E}^{(m-1)} \chi\right)-\chi \cdot \chi H_{E}^{(m-1)} \chi \\
&+i\left\{\chi H\left(\chi H_{E}^{(m-1)} \chi\right)+H \chi \cdot \chi H_{E}^{(m-1)} \chi\right\} .
\end{aligned}
$$

Hence (28) $\left(g=\chi, h=\chi H_{E}^{(m-1)} \chi\right)$ shows that

$$
\lim _{\eta \downarrow 0}\left\{u_{m+1}(\cdot+i \eta)+v_{m+1}(\cdot+i \eta)-u_{1}(\cdot+i \eta) u_{m}(\cdot+i \eta)\right\}=0
$$

on $\mathbb{R}$. In particular, this holds on $\mathbb{R}-E$. Hence, by the theorem of identity, $u_{m+1}(z)+v_{m+1}(z)-u_{1}(z) u_{m}(z)=0$. Equality (29) shows that $v_{m+1}(z)=m u_{m+1}(z)+(m-1) u_{m-1}(z)$. Thus

$$
(m+1) u_{m+1}(z)+(m-1) u_{m-1}(z)-u_{1}(z) u_{m}(z)=0 \quad(m \geq 1),
$$

which yields that

$$
\begin{gathered}
\sum_{m=0}^{\infty} m t^{m} u_{m}(z)+t^{2} \sum_{m=0}^{\infty} m t^{m} u_{m}(z)=t u_{1}(z) \sum_{m=0}^{\infty} t^{m} u_{m}(z), \quad \text { i.e. } \\
t \frac{\partial}{\partial t} P_{t}(z)+t^{3} \frac{\partial}{\partial t} P_{t}(z)=t u_{1}(z) P_{t}(z)
\end{gathered}
$$

This is the required equality (31). 
We can choose $x_{0} \in \mathbb{R}-E, \eta>0$ so that $P_{t}(x)>0, u_{1}(x)>0$ for all $x \in\left(x_{0}-\eta, x_{0}+\eta\right), 0<t<1$. Equality (31) shows that $\frac{1}{1+t^{2}} u_{1}(x)=\frac{\partial}{\partial t} P_{t}(x) / P_{t}(x) \quad\left(x \in\left(x_{0}-\eta, x_{0}+\eta\right), 0<t<1\right)$, which gives that

$$
P_{t}(x)=\exp \left\{\int_{0}^{t} \frac{d s}{1+s^{2}} u_{1}(x)\right\} \quad\left(x \in\left(x_{0}-\eta, x_{0}+\eta\right), 0<t<1\right)
$$

because $P_{0}=1$. By the theorem of identity,

$$
P_{t}(z)=\exp \left\{\int_{0}^{t} \frac{d s}{1+s^{2}} u_{1}(z)\right\} \quad(0<t<1) .
$$

Since $P_{t}(z)$ and $\exp \left\{\left(\int_{0}^{t}\left(d s /\left(1+s^{2}\right)\right) u_{1}(z)\right\}\right.$ are analytic in the unit disk as functions of $t$, this equality holds for $-1<t<0$ also. Thus

$$
\begin{aligned}
1+\frac{1}{\pi} \int_{E} \frac{h_{E}(s)}{s-z} d s=1+\frac{1}{\pi} \int_{E} \frac{1}{s-z} \sum_{l=1}^{\infty} H_{E}^{(2 l-1)} \chi(s) d s \\
=\lim _{t \uparrow 1} \sum_{l=0}^{\infty} t^{2 l} u_{2 l}(z)=\frac{1}{2} \lim _{t \uparrow 1}\left\{P_{-t}(z)+P_{t}(z)\right\} \\
=\frac{1}{2}\left\{\exp \left(-\frac{\pi}{4} u_{1}(z)\right)+\exp \left(\frac{\pi}{4} u_{1}(z)\right)\right\}, \\
-\frac{1}{\pi} \int_{E} \frac{d s}{s-z}-\frac{1}{\pi} \int_{E} \frac{H_{E}^{(1)} h_{E}(s)}{s-z} d s=\frac{1}{2} \lim _{t \uparrow 1}\left\{P_{-t}(z)-P_{t}(z)\right\} \\
=\frac{1}{2}\left\{\exp \left(-\frac{\pi}{4} u_{1}(z)\right)-\exp \left(\frac{\pi}{4} u_{1}(z)\right)\right\},
\end{aligned}
$$

which gives (27).

\section{REFERENCES}

[1] A. P. Calderón, Cauchy integrals on Lipschitz curves and related operators, Proc. Nat. Acad. Sci. USA, 74 (1977), 1324-1327.

[2] A. M. Davie, Analytic capacity and approximation problems, Trans. Amer. Math. Soc., 171 (1972), 409-444.

[3] A. Denjoy, Sur les fonctions analytiques uniformes à singularités discontinues, C. R. Acad. Sci. Paris, 149 (1909), 258-260.

[4] J. Garnett, Analytic Capacity and Measure, Lecture Notes in Mathematics, Vol. 297, Springer-Verlag, Berlin-Heidelberg-New York, 1972.

[5] P. W. Jones, Square functions, Cauchy integrals, analytic capacity, and harmonic measure, preprint.

[6] N. S. Landkof, Foundations of Modern Potential Theory, Springer-Verlag, BerlinHeidelberg-New York, 1972. 
[7] B. B. Mandelbrot, The Fractal Geometry and Nature, Freeman, San Francisco, 1982.

[8] D. E. Marshall, Removable sets for bounded analytic functions, in Linear and Complex Analysis Problem Book (Edited by V. P. Havin, S. V. Hruščëv and N. K. Nikol' skiī), Lecture Notes in Mathematics, Vol. 1043, Springer-Verlag, Berlin-Heidelberg-New York-Tokyo, 1984, 485-490.

[9] T. Murai, Comparison between analytic capacity and the Buffon needle probability, Trans. Amer. Math. Soc., 304 (1987), 501-514.

[10] __ A Real Variable Method for the Cauchy Transform, and Analytic Capacity, Lecture Notes in Mathematics, Vol. 1307, Springer-Verlag, Berlin-HeidelbergNew York-London-Paris-Tokyo, 1988.

[11] Ch. Pommerenke, Über die analytische Kapazität, Arch. Math., 11 (1960), 270277.

[12] A. G. Vitushkin, Example of a set of positive length but of zero analytic capacity, (Russian) Dokl. Akad. Nauk SSSR, 127 (1959), 246-249.

Received July 21, 1988.

NAGOYA UNIVERSITY

CHIKUSA-KU, NAGOYA, 464

JAPAN 


\section{PACIFIC JOURNAL OF MATHEMATICS EDITORS}

\author{
V. S. VARADARAJAN \\ (Managing Editor) \\ University of California \\ Los Angeles, CA 90024-1555-05 \\ Herbert Clemens \\ University of Utah \\ Salt Lake City, UT 84112 \\ ThOMAs ENRIGHT \\ University of California, San Diego \\ La Jolla, CA 92093
}

R. FINN

Stanford University

Stanford, CA 94305

HeRmanN FlaschKa

University of Arizona

Tucson, AZ 85721

VAUGHAN F. R. JoNES

University of California

Berkeley, CA 94720

SteVen KerCKhofF

Stanford University

Stanford, CA 94305
ROBION KIRBY

University of California

Berkeley, CA 94720

C. C. Moore

University of California

Berkeley, CA 94720

HAROLD STARK

University of California, San Diego

La Jolla, CA 92093

\section{ASSOCIATE EDITORS}
R. ARENS
E. F. BECKENBACH
B. H. NeumanN
F. Wolf
K. YoshidA
(1906-1982)
(1904-1989)

\section{SUPPORTING INSTITUTIONS}

UNIVERSITY OF ARIZONA
UNIVERSITY OF BRITISH COLUMBIA
CALIFORNIA INSTITUTE OF TECHNOLOGY
UNIVERSITY OF CALIFORNIA
MONTANA STATE UNIVERSITY
UNIVERSITY OF NEVADA, RENO
NEW MEXICO STATE UNIVERSITY
OREGON STATE UNIVERSITY
UNIVERSITY OF ARIZONA
UNIVERSITY OF BRITISH COLUMBIA
UNIVERSITY OF CALIFORNIA
MONTANA STATE UNIVERSITY
NEW MEXICO STATE UNIVERSITY

\author{
UNIVERSITY OF OREGON \\ UNIVERSITY OF SOUTHERN CALIFORNIA \\ STANFORD UNIVERSITY \\ UNIVERSITY OF HAWAII \\ UNIVERSITY OF TOKYO \\ UNIVERSITY OF UTAH \\ WASHINGTON STATE UNIVERSITY \\ UNIVERSITY OF WASHINGTON
}

The Supporting Institutions listed above contribute to the cost of publication of this Journal, but they are not owners or publishers and have no responsibility for its content or policies.

Mathematical papers intended for publication in the Pacific Journal of Mathematics should be in typed form or offset-reproduced (not dittoed), double spaced with large margins. Please do not use built up fractions in the text of the manuscript. However, you may use them in the displayed equations. Underline Greek letters in red, German in green, and script in blue. The first paragraph must be capable of being used separately as a synopsis of the entire paper. In particular it should contain no bibliographic references. Please propose a heading for the odd numbered pages of less than 35 characters. Manuscripts, in triplicate, may be sent to any one of the editors. Please classify according to the 1980 Mathematics Subject Classification (1985 Revision) scheme which can be found in the December index volumes of Mathematical Reviews. Supply name and address of author to whom proofs should be sent. All other communications should be addressed to the managing editor, or Elaine Barth, University of California, Los Angeles, California 90024-1555-05.

There are page-charges associated with articles appearing in the Pacific Journal of Mathematics. These charges are expected to be paid by the author's University, Government Agency or Company. If the author or authors do not have access to such Institutional support these charges are waived. Single authors will receive 50 free reprints; joint authors will receive a total of 100 free reprints. Additional copies may be obtained at cost in multiples of 50 .

The Pacific Journal of Mathematics is issued monthly as of January 1966. Regular subscription rate: $\$ 190.00$ a year (6 Vols., 12 issues). Special rate: $\$ 95.00$ a year to individual members of supporting institutions.

Subscriptions, orders for numbers issued in the last three calendar years, and changes of address should be sent to Pacific Journal of Mathematics, P.O. Box 969, Carmel Valley, CA 93924, U.S.A. Old back numbers obtainable from Kraus Periodicals Co., Route 100, Millwood, NY 10546.

The Pacific Journal of Mathematics at P.O. Box 969, Carmel Valley, CA 93924 (ISSN 0030-8730) is published monthly. Second-class postage paid at Carmel Valley, California 93924, and additional mailing offices. Postmaster: send address changes to Pacific Journal of Mathematics, P.O. Box 969, Carmel Valley, CA 93924.

\section{PUBLISHED BY PACIFIC JOURNAL OF MATHEMATICS, A NON-PROFIT CORPORATION}




\section{Pacific Journal of Mathematics}

\section{Vol. 143, No. $2 \quad$ April, 1990}

Gustavo Corach, Horacio Porta and Lázaro Recht, Differential geometry of systems of projections in Banach algebras ................. 209

Peter Fleischmann and Jens Carsten Jantzen, Simple periodic modules of

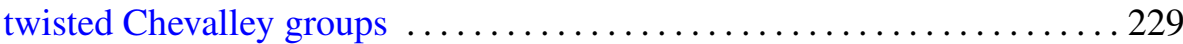

Niels Gronbaek, Amenability of discrete convolution algebras, the commutative case ...................................243

Nguyên H. V. Hung, The mod 2 equivariant cohomology algebras of

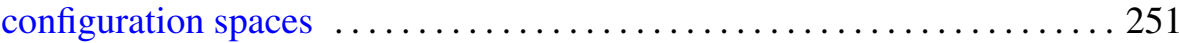

Wojciech Kucharz, Global almost analytic algebraicity of analytic sets . . . 287 John Merrill, A class of consistent anti-Martin's axioms .............. 301

Takafumi Murai, The power 3/2 appearing in the estimate of analytic

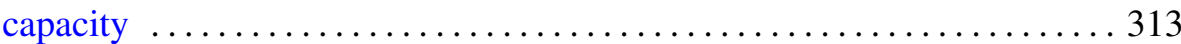

L. Panaitopol and Doru Stefanescu, On the generalized difference

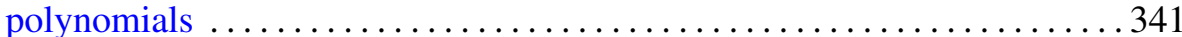

Katsuhiro Shiohama, Takashi Shioya and Minoru Tanaka, Mass of rays

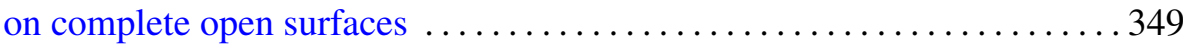

Gerhard Ströhmer, About compressible viscous fluid flow in a bounded

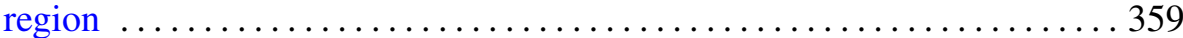

A. Ülger, Arens regularity sometimes implies the RNP 